\title{
Theoretical development of a stiffness decomposition method for calculating the displacement of complex bending structures
}

\author{
Yuguo Zheng*, Xiangshou Xiao, Minghang Chen, Yu Wang and Yingliang Song \\ School of Civil Engineering, Hunan University of Science and Technology, Xiangtan 411201, PR China \\ * Corresponding Author: ygzheng@hnust.edu.cn
}

Submitted : 24/02/2020

Revised : 30/06/2020

Accepted :09/07/2020

\begin{abstract}
It is very difficult to provide analytical displacement solutions for complex bending structures, such as beams with variable cross-sections, in structural analysis. The common methods used for such analysis - the direct integration method and the conventional graph multiplication method - have disadvantages of inefficiency and large computational costs. Therefore, a new approach called the stiffness decomposition method was proposed to overcome these shortcomings. The fundamental principle of this new approach was derived based on the unit load method. The general calculation equation of displacement was derived and provided for general n-segment complex bending structures, and an operational procedure for this method was constructed to facilitate its application. Then, the method was applied to two case studies involving classic complex bending structures. The results showed the correctness and effectiveness of the proposed method. The stiffness decomposition method was simpler and more efficient than the other two methods: the number of computations required by the stiffness decomposition method accounted for only $47.4 \%$ to $84.0 \%$ of the number of computations required by the other methods in the two case studies. The clear mathematical and mechanical derivation of the proposed method makes it easy to understand. Furthermore, the simplicity and practicality of this method make it extensively applicable.
\end{abstract}

Keywords: Complex bending structure; Displacement calculation; Direct integration method; Graph multiplication method; Stiffness decomposition method; Unit load method.

\section{INTRODUCTION}

Complex bending structures are widely utilized in macromechanical and civil engineering applications (Ren et al., 2019 \& Huang et al., 2019). Many studies focus on the structural analysis and the displacement calculation of them both from the static perspective and the dynamic one. The locking-free isogeometric analysis of complex threedimensional beam structures was investigated by Xia and Liao (2018) by modeling the beam structure with multipatch nonuniform rational B-spline functions. The technique of multiple sets of approximation functions originated from quasi-conforming finite element method was used to solve the locking problem. Tang et al. (2019) deduced the theoretical formula of deflection curvature of beam structure under uniform load to identify the damage 
location of the structure based on the force method. The span-by-span uniformly loaded deflection curvature index was proposed to solve the problem of missing damage identification for uniformly loaded continuous beam.

Ma et al. (2020) put forward a displacement reconstruction method for slender flexible beam based on polynomial fitting. The accuracy and feasibility of the method were validated by finite element simulation and reconstruction of experimental data for linear and nonlinear vibrations of slender flexible beams. A new technique was proposed by Wang et al. (2017) to estimate the dynamic deflection of beam structures based on strain modes. The method was verified by numerical simulations and experimental tests.

It can be shown that the above methods are effective to provide numerical solutions for complex beam structures for different purposes under different conditions.

On microcomplex beam structures, some researchers have completed studies and developed some meaningful achievements, especially in nano-scaled beam modeling and analyzing. Civalek and Demir (2011) developed the elastic beam model by using nonlocal elasticity theory for the bending analysis of microtubules based on the EulerBernoulli beam theory. The model was applied to study the static analysis of microtubules using the method of differential quadrature. Detailed numerical analyses about the effects of boundary conditions and load types were conducted and the influence of nonlocal parameter on the static response of microtubules was discussed.

Free vibration analysis of functionally graded (FG) size-dependent nanobeams was conducted by Eltaher et al. (2012) based on FEM. The nanobeam was modeled according to Euler-Bernoulli beam theory and its equations of motion were derived using Hamilton's principle. The finite element method was used to discretize the model and obtain a numerical approximation of the equation of motion. Demir and Civalek (2013) investigated the size-effects in the torsional and axial response of microtubules by using the nonlocal continuum rod model. A finite element procedure was used for modeling and solution of nonlocal discrete system equation of microtubules. The influence of the small length scale on the vibration frequencies was examined by torsional and axial vibration cases. The longitudinal free vibration problem of a microscaled bar was studied by Akgöz and Civalek (2014) based on the strain gradient elasticity theory. The equation of motion with initial conditions, classical and nonclassical corresponding boundary conditions for a microscaled elastic bar was derived via Hamilton's principle. Civalek et al. (2020) performed the free vibration analyses of embedded carbon and silica carbide nanotubes lying on an elastic matrix based on Eringen's nonlocal elasticity theory. The obtained vibration equations of motions were solved by the method of separation of variables and finite element-based Hermite polynomials for various boundary conditions. Parametric analyses were conducted to discuss the effects of boundary conditions, system modeling, structural sizes such as length, cross-sectional sizes, elastic matrix, mode number, and nonlocal parameters on the natural frequencies of these nanostructures.

Civalek and Demir (2016) proposed a simple nonlocal beam model to study buckling response of protein microtubules. Finite element procedure was used for solution of nonlocal differential equation of microtubules for elastic stability.

It shows that the above studies on microcomplex beam structures focus on the mechanical property analyses for microtubules based on nano-scaled beam modeling. Extensive numerical simulation and finite element analyses were conducted based on the modeling technology. Different factors, such as material distribution profile, nonlocal effect elastic modulus, material properties, boundary conditions and size-effect, were investigated to explore the influence on the bending, torsional and axial response, free vibration and elastic stability of microtubules. The results present some benchmarks in the field.

With the rapid development of finite element technology, numerical solutions of structural analysis are very easy to obtain, which makes the large-scale numerical simulation possible. Large-scale numerical calculation can 
provide amount of data and make it easy to find out the implied laws. It is also usually applied to the displacement calculation of complex bending structures and the advantage is significant. The above literatures on the analyses of macro- and microbeam structures also present the same idea.

However, analytical methods still have a substantial impact on the development of structural analysis (Shvab'yuk et al., 2016). Analytical approaches usually contain clear mechanical concepts and profound mechanical principles. They facility to provide elastic estimation rapidly with enough accuracy and small computation costs. And analytical methods influence the development of structural analysis deeply. So, it is important and necessary to promote the study of analytical methods in structural analysis.

It is known that the determination of structural displacements is an important aspect of analyzing the load response of engineering structures. The structural displacement is related to the stiffness check of the structure and the performance of the structure in the normal service state (Zeng et al., 2019). Large displacements could cause a structure to function improperly or even to collapse. In addition, the computation of displacements for statically determinate structures provides a foundation for analyzing the displacements of statically indeterminate structures (Illouli et al., 2019). Therefore, displacement calculations play an important role in classic structural analysis.

The most common analytical methods for calculating structural displacements include the direct integration method (DIM) and the conventional graph multiplication method (CGM). In classic structural analysis, the operation of the DIM and the CGM is based on the unit load method, which is derived by the virtual work principle of deformable structural systems (Zhang et al., 2018). The displacements of bending structures are mainly caused by bending moments under loads. Therefore, the minor displacements caused by axial and shear forces are always neglected (Ghali et al., 2017).

The basic operation of the DIM for a bending structure includes exerting a unit virtual load $\mathrm{P}=1$ in the direction of the undetermined displacement at one specified point of the structure, solving the bending moments $\bar{M}(x)$ and $M_{P}(x)$ caused by the unit load and actual loads, respectively, integrating the two sets of bending moment functions along the length $\mathrm{L}$ of each member, and superimposing the integration results of all the members (Sun et al., 2017). Then, the desired displacement can be given as

$$
\Delta=\sum \int_{0}^{L} \frac{\bar{M}(x) M_{P}(x)}{E I(x)} \mathrm{d} s
$$

where $E$ is the elastic modulus of the materials, $I(x)$ is the inertial moment of the cross section of a member, $E I(x)$ represents the flexural rigidity of a member cross section, and $d s$ is the segmental differential length of a member. Equation (1) is the general equation of the DIM. Vereshchagin proposed the CGM based on the DIM in 1925 (Karnovsky et al., 2017). The application of this method requires three basic conditions: (a) the members of the structure are straight bars, (b) the flexural stiffness $E I(x)$ of every element is constant or piecewise constant along its length, and (c) at least one of the $\bar{M}(x)$ and $M_{P}(x)$ diagrams is a linear or piecewise linear graph.In structural analysis, the areas and centroids of some commonly used bending moment graphs can be easily determined, as shown in Table 1. In these standard bending moment graphs, $L$ is the length of a segment or an element, $h$ represents the height of the bending moment diagram, and $C$ indicates the centroid of the graph. 
Table 1. Areas and centroids of standard bending moment graphs.

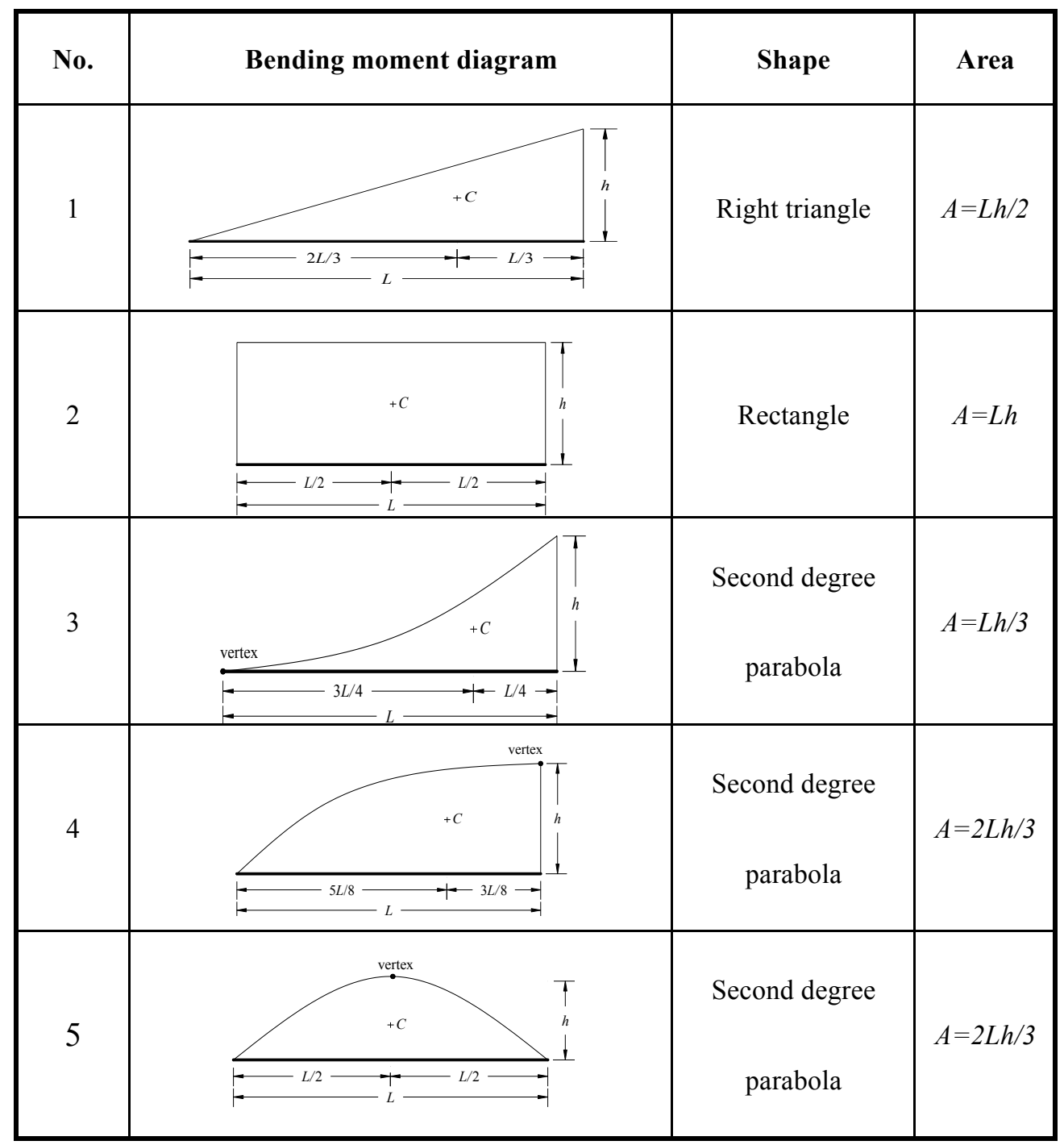

If a structure satisfies the above three basic conditions, the CGM includes four operation steps to provide structural displacements based on the unit load method: (a) compute the area $A_{i}$ of one bending moment graph for every segment of the structure, (b) calculate the vertical coordinate $y_{i}$ of the other linear bending moment diagram at the location corresponding to the centroid of the previous diagram, (c) multiply $A_{i}$ and $y_{i}$ for every segment or member, and (d) superpose the multiplication results of all segments or members. Then, the displacement $\Delta$ at one specified point of the bending structure can be written as

$$
\Delta=\sum \int_{0}^{L} \frac{\bar{M}(x) M_{P}(x)}{E I(x)} \mathrm{d} s=\sum \int_{0}^{L} \frac{\bar{M}(x) M_{P}(x)}{E I(x)} \mathrm{d} x=\sum \frac{A_{i} y_{i}}{(E I)_{i}}
$$


The CGM is more straightforward and convenient than the DIM for simple bending structure systems. Moreover, the CGM is very easy to understand, and the corresponding computational cost is relatively small. Therefore, this method has been applied in engineering practice to some degree (Su et al., 2013 \& Zhang et al., 2015).

However, the application of the DIM is very complicated, and the calculation is tedious and time consuming for many complex bending structures, such as variable-section beams or stepped beams. When the CGM is used to compute the displacements of these structures, the bending moment graphs must be divided into many partitions. The number of graph multiplications is excessive because of the complexity of the structure. Therefore, the application of the CGM in these complex bending structures is still very complicated, which limits its relative advantage over the DIM (Zheng et al., 2019 \& Huang et al., 2016).

Therefore, an innovative method named the stiffness decomposition method (SDM) is proposed and developed in this paper. It is a new analytical approach for displacement calculation of complex bending structures. This method can overcome the difficulties faced by the DIM and the CGM in determining the displacements of these complex structures. The proposed approach enables simpler, more efficient and more practical displacement computations and simultaneously further develops the comparative advantages of the CGM over the DIM. It is expected that the proposed method will promote the development of analytical methods in structural analysis further.

\section{STIFFNESS DECOMPOSITION METHOD}

\section{Basic Assumptions}

This paper focuses on displacement calculations for linearly deformable systems or structures. The theoretical foundations of the calculations are the virtual work principle and the unit load method (Bao et al., 2008).

The term linearly deformable structures implies that the displacements of these structures are proportional to the loads applied on them. Hence, when the loads are removed, the displacements disappear. To be considered a linearly deformable structure, the following conditions must be satisfied:

(a) The materials of the structure are linearly elastic, i.e., the relationship between stress and strain is linear.

(b) The deformation of the structure is sufficiently small to neglect the changes from the original loading effects.

(c) A linearly deformable structure is also called a linearly elastic structure. The principle of superposition can be applied to this kind of structure. Therefore, the computation of displacements of a linearly deformable structure can be implemented by using the principle of superposition.

\section{Theoretical Derivation}

A general $n$-segment complex bending structure, as shown in Figure 1, is used to derive the basic principle of the SDM. This structure is composed of $n$ straight segments, which are denoted segment $\# 1$ through segment $\# n$. For this structure, the axis is linear, and the total length is $L$. In Figure $1, B_{1} \ldots \ldots B_{n+l}$ represent the demarcation points between the segments, $L_{1} \ldots \ldots L_{n}$ are the lengths of every section, and $m_{l} E I \ldots \ldots m_{n} E I$ express the flexural rigidities of every segment. 


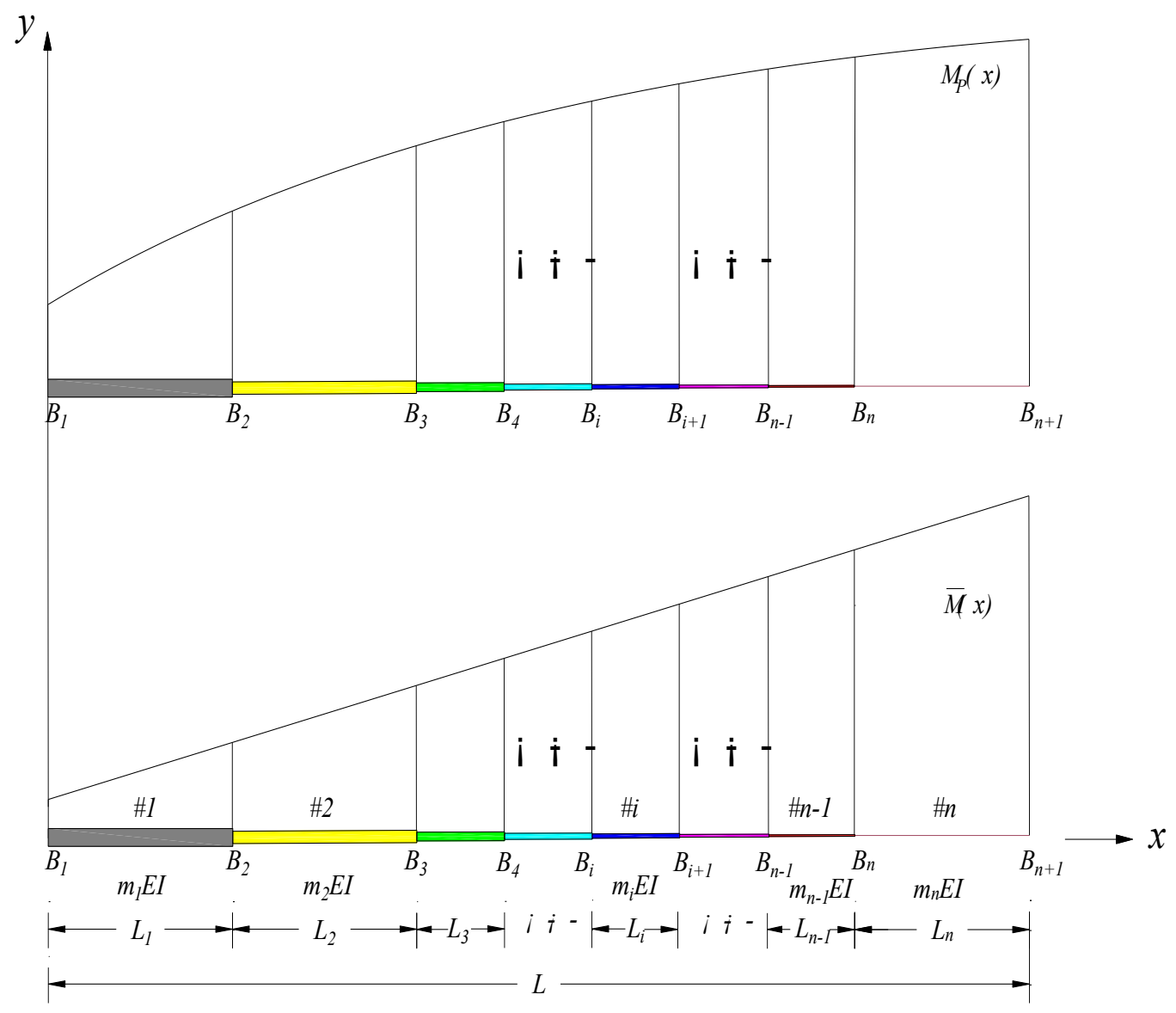

Figure 1. General $n$-segment complex structure and bending moment $\bar{M}(x)$ and $M_{P}(x)$ graphs.

It is assumed that the displacement at one specified point of the structure in Figure 1 should be solved under actual loads. According to the basic operation of the unit load method, the bending moment functions $\bar{M}(x)$ and $M_{P}(x)$ should be given first. We assume that the two functions have been determined, and their diagrams are illustrated in Figure 1. Based on the requirements of the CGM, one of the $\bar{M}(x)$ and $M_{P}(x)$ diagrams is linear. Therefore, it is assumed that the bending moment $\bar{M}(x)$ graph is linear here. As shown in Figure 1, the coordinate origin is located at point $B_{l}$. The $x$ axis is the axis of the structure whose direction is right positive. The $y$ axis is perpendicular to the $x$ axis, for which the direction is positive upward.

Because the segments differ in flexural rigidity, piecewise integration should be conducted along the length of the structural axis to compute the displacement with the DIM. If $d_{s}=d_{x}$ is substituted into Equation (1), we obtain 


$$
\begin{aligned}
\Delta & =\sum \int_{0}^{L} \frac{\bar{M}(x) M_{P}(x)}{E I(x)} \mathrm{d} s \\
& =\int_{0}^{L_{1}} \frac{\bar{M}(x) M_{P}(x)}{m_{1} E I} \mathrm{~d} x+\int_{L_{1}}^{L_{1}+L_{2}} \frac{\bar{M}(x) M_{P}(x)}{m_{2} E I} \mathrm{~d} x++\int_{L_{1}+L_{2}}^{L_{1}+L_{2}}+L_{\mathrm{n}-1}^{+L_{\mathrm{n}}} \frac{\bar{M}(x) M_{P}(x)}{m_{n} E I} \mathrm{~d} x \\
& =\int_{0}^{L_{1}} \frac{\bar{M}(x) M_{P}(x)}{m_{1} E I} \mathrm{~d} x+\sum_{i=2}^{n}\left(\int_{L_{1}+L_{2}}^{L_{1}+L_{2}}+L_{\mathrm{i}-1} \frac{\bar{M}(x) M_{P}(x)}{m_{i} E I} \mathrm{~d} x\right)
\end{aligned}
$$

The piecewise integration will be very complicated if there are many segments with different flexural rigidities.

On the other hand, the CGM can be implemented to compute the displacement of the general $n$-segment bending structure if the three conditions are satisfied. The piecewise graph multiplication must be conducted by combining Equations. (2) and (3). However, utilizing only one graph multiplication operation for a segment cannot directly give its part of the total displacement solution because the bending moment $M_{P}(x)$ graph of this segment is not a standard graph (Wu et al., 2017). The $M_{P}(x)$ graph of this segment must be divided into blocks again to make every block a standard block. Therefore, the division process for the segments and blocks will make the whole graph multiplication of the complex bending structure very complicated.

To simplify the difficult computations described above, we hierarchically decompose Equation (3) in accordance with the division of flexural stiffness in the segment; the corresponding expression is shown in

$$
\begin{aligned}
& \Delta=\int_{0}^{L_{1}} \frac{\bar{M}(x) M_{P}(x)}{m_{1} E I} \mathrm{~d} x+\int_{L_{1}}^{L_{1}+L_{2}} \frac{\bar{M}(x) M_{P}(x)}{m_{2} E I} \mathrm{~d} x++\int_{L_{1}+L_{2}+L_{n-1}}^{L_{1}+L_{2}} \frac{\overline{L_{n}}}{m_{n} E I} \mathrm{M}(x) M_{P}(x) \mathrm{d} x
\end{aligned}
$$

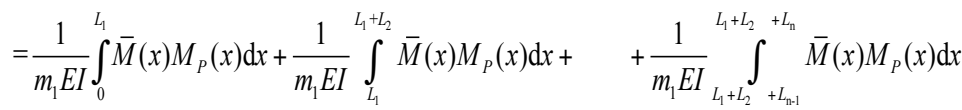

$$
\begin{aligned}
& +\left(\frac{1}{m_{2} E I}-\frac{1}{m_{1} E I}\right) \int_{L_{1}}^{L_{1}+L_{2}} \bar{M}(x) M_{P}(x) \mathrm{d} x+\quad+\left(\frac{1}{m_{n} E I}-\frac{1}{m_{1} E I}\right)_{L_{1}+L_{2}+L_{n-1}}^{L_{1}+L_{2}}+L_{L_{n}} \bar{M}(x) M_{P}(x) \mathrm{d} x \\
& =\frac{1}{m_{1} E I} \int_{0}^{L} \bar{M}(x) M_{P}(x) \mathrm{d} x+\frac{m_{1}-m_{2}}{m_{1} m_{2} E I} \int_{L_{1}}^{L_{1}+L_{2}} \bar{M}(x) M_{P}(x) \mathrm{d} x+\frac{m_{1}-m_{3}}{m_{1} m_{3} E I} \int_{L_{1}+L_{2}}^{L_{1}+L_{2}+L_{3}} \bar{M}(x) M_{P}(x) \mathrm{d} x+\quad+\frac{m_{1}-m_{n}}{m_{1} m_{n} E I} \int_{L_{1}+L_{2}+L_{2}}^{L_{1}+L_{2}}+L_{L_{n-1}}^{+L_{n}} \bar{M}(x) M_{P}(x) \mathrm{d} x
\end{aligned}
$$

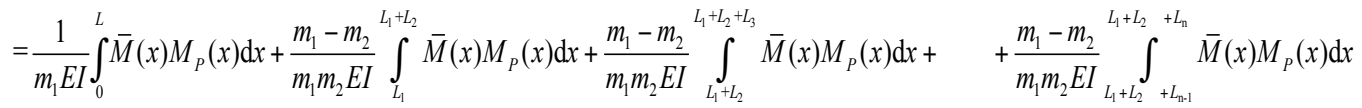

$$
\begin{aligned}
& +\left(\frac{m_{1}-m_{3}}{m_{1} m_{3} E I}-\frac{m_{1}-m_{2}}{m_{1} m_{2} E I}\right) \int_{L_{1}+L_{2}}^{L_{1}+L_{2}+L_{3}} \bar{M}(x) M_{P}(x) \mathrm{d} x+\quad+\left(\frac{m_{1}-m_{n}}{m_{1} m_{n} E I}-\frac{m_{1}-m_{2}}{m_{1} m_{2} E I}\right)_{L_{1}+L_{2}}^{L_{1}+L_{2}+L_{n-1}} \sum_{L_{n}} \bar{M}(x) M_{P}(x) \mathrm{d} x \\
& =\frac{1}{m_{1} E I} \int_{0}^{L} \bar{M}(x) M_{P}(x) \mathrm{d} x+\frac{m_{1}-m_{2}}{m_{1} m_{2} E I} \int_{L_{1}}^{L} \bar{M}(x) M_{P}(x) \mathrm{d} x \\
& +\frac{m_{2}-m_{3}}{m_{2} m_{3} E I} \int_{L_{1}+L_{2}}^{L_{1}+L_{2}+L_{3}} \bar{M}(x) M_{P}(x) \mathrm{d} x+\quad+\frac{m_{2}-m_{n}}{m_{2} m_{n} E I} \int_{L_{1}+L_{2}}^{L_{1}+L_{2}}+L_{n-1}+L_{n} \quad \bar{M}(x) M_{P}(x) \mathrm{d} x \\
& = \\
& =\frac{1}{m_{1} E I} \int_{0}^{L} \bar{M}(x) M_{P}(x) \mathrm{d} x+\frac{m_{1}-m_{2}}{m_{1} m_{2} E I} \int_{L_{1}}^{L} \bar{M}(x) M_{P}(x) \mathrm{d} x+\frac{m_{2}-m_{3}}{m_{2} m_{3} E I} \int_{L_{1}+L_{2}}^{L} \bar{M}(x) M_{P}(x) \mathrm{d} x+\quad+\frac{m_{n-1}-m_{n}}{m_{n-1} m_{n} E I} \int_{L_{1}+L_{2}+L_{n-1}}^{L} \bar{M}(x) M_{P}(x) \mathrm{d} x
\end{aligned}
$$




$$
\Delta=\frac{1}{m_{1} E I} \int_{0}^{L} \bar{M}(x) M_{P}(x) \mathrm{d} x+\sum_{i=2}^{n}\left(\frac{m_{i-1}-m_{i}}{m_{i-1} m_{i} E I} \int_{L_{1}+L_{2}}^{L} \bar{M}(x) M_{P}(x) \mathrm{d} x\right)
$$

The derivation of Equation (4) and the composition of Equation (5) were analyzed carefully. It was found that every component of the displacement $\Delta$ in Equation (5) was derived from the decomposition of every component in Equation (3). Then, the decomposed components were superposed based on the division of flexural stiffness in the segment. The aim of the decomposition in Equation (4) was to make the flexural rigidity of every component in Equation (5) constant along the length of this segment. The bending moment $M_{P}(x)$ of this segment was transformed into a standard graph to a large extent. Therefore, we can avoid tedious graph multiplication due to complicated segment and block division, and the whole displacement computation can be greatly simplified. Correspondingly, $m_{1} E I$ and $m_{i-1} m_{i} E I /\left(m_{i-1}-m_{i}\right)$ were named the $1^{s t}$ and $i^{\text {th }}$ postdecomposition stiffness, respectively. After the stiffness decomposition, the reciprocals of these decomposed stiffnesses were applied to calculate the corresponding displacements with the CGM. All the displacements were superposed to supply the final displacement of the specified point.

We defined each component in Equation (5) as equal to $\Delta_{i}$, which corresponds to each postdecomposition stiffness, as shown in Equation (6). Note that $\Delta_{i}$ is defined as the displacement of the general $n$-segment bending structure in the $i^{\text {th }}$ state of stiffness decomposition.

$$
\left\{\begin{array}{l}
\Delta_{1}=\frac{1}{m_{1} E I} \int_{0}^{L} \bar{M}(x) M_{P}(x) \mathrm{d} x \\
\Delta_{2}=\frac{m_{1}-m_{2}}{m_{1} m_{2} E I} \int_{L_{1}}^{L} \bar{M}(x) M_{P}(x) \mathrm{d} x \\
\Delta_{3}=\frac{m_{2}-m_{3}}{m_{2} m_{3} E I} \int_{L_{1}+L_{2}}^{L} \bar{M}(x) M_{P}(x) \mathrm{d} x \\
\Delta_{i}=\frac{m_{i-1}-m_{i}}{m_{i-1} m_{i} E I} \int_{L_{1}+L_{2}}^{L} \bar{M}(x) M_{P}(x) \mathrm{d} x \\
\Delta_{n-1}=\frac{m_{n-2}-m_{n-1}}{m_{n-2} m_{n-1} E I} \int_{L_{1}+L_{2}}^{L}+L_{\mathrm{n}-2} \\
\Delta_{n}=\frac{m_{n-1}-m_{n}}{m_{n-1} m_{n} E I} \int_{L_{1}+L_{2}}^{L} \bar{M}(x) M_{P}(x) \mathrm{d} x
\end{array}\right.
$$

In addition, Equation (6) is translated as 


$$
\begin{aligned}
& \Delta_{1}=\frac{1}{m_{1} E I} \int_{0}^{L} \bar{M}(x) M_{P}(x) \mathrm{d} x \\
& \Delta_{2}=\frac{m_{1}-m_{2}}{m_{1} m_{2} E I} \int_{L_{1}}^{L} \bar{M}(x) M_{P}(x) \mathrm{d} x=\frac{1}{\infty E I} \int_{0}^{L_{1}} \bar{M}(x) M_{P}(x) \mathrm{d} x+\frac{m_{1}-m_{2}}{m_{1} m_{2} E I} \int_{L_{1}}^{L} \bar{M}(x) M_{P}(x) \mathrm{d} x \\
& \Delta_{3}=\frac{m_{2}-m_{3}}{m_{2} m_{3} E I} \int_{L_{1}+L_{2}}^{L} \bar{M}(x) M_{P}(x) \mathrm{d} x=\frac{1}{\infty E I} \int_{0}^{L_{1}+L_{2}} \bar{M}(x) M_{P}(x) \mathrm{d} x+\frac{m_{2}-m_{3}}{m_{2} m_{3} E I} \int_{L_{1}+L_{2}}^{L} \bar{M}(x) M_{P}(x) \mathrm{d} x \\
& \Delta_{i}=\frac{m_{i-1}-m_{i}}{m_{i-1} m_{i} E I} \int_{L_{1}+L_{2}}^{L} \bar{M}(x) M_{i-1}(x) \mathrm{d} x=\frac{1}{\infty E I} \int_{0}^{L_{1}+L_{2}} \bar{M}(x) M_{P}(x) \mathrm{d} x+\frac{m_{i-1}-m_{i}}{m_{i-1} m_{i} E I} \int_{L_{1}+L_{2}}^{L} \bar{M}+L_{i-1}(x) M_{P}(x) \mathrm{d} x
\end{aligned}
$$

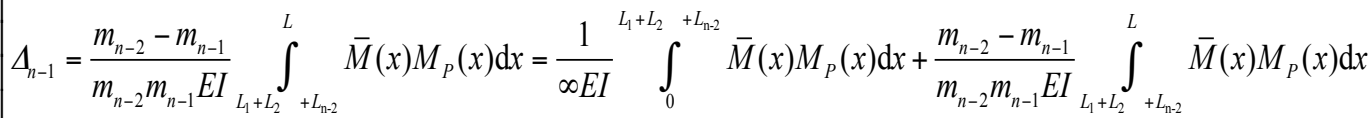

$$
\begin{aligned}
& \Delta_{n}=\frac{m_{n-1}-m_{n}}{m_{n-1} m_{n} E I} \int_{L_{1}+L_{2}}^{L} \bar{M}(x) L_{n-1}(x) \mathrm{d} x=\frac{1}{\infty E I} \int_{0}^{L_{1}+L_{2}} \int_{L_{n-1}}^{+} \bar{M}(x) M_{P}(x) \mathrm{d} x+\frac{m_{n-1}-m_{n}}{m_{n-1} m_{n} E I} \int_{L_{1}+L_{2}}^{L} \bar{M}(x) M_{n-1}(x) \mathrm{d} x
\end{aligned}
$$

In contrast to Equation (6), one displacement component is added to $\Delta_{2}, \Delta_{3} \ldots \ldots \Delta_{n}$ in Equation (7). The corresponding integration of the displacement component is in accordance with the left length of the structure, and the corresponding stiffness is $\infty E I$. Because the stiffness is $\infty E I$, the corresponding displacement is zero. Therefore, the equivalence of every equation in Equation (7) still holds. The purpose of this procedure is to ensure that every displacement $\Delta_{i}$ in Equation (7) corresponds to the total length of the structure and to the whole bending moment graph of the structure. After this process, the principle of the new method can be rationally and easily understood. Hence, the new method can be conveniently applied for the following graph multiplication computation.

Based on the derivation of Equation (7), $\Delta_{1}, \Delta_{2}, \Delta_{3}, \Delta_{i}, \Delta_{n-1}$, and $\Delta_{n}$ can be computed by graph multiplication, as illustrated in Figure 2 through Figure 7.

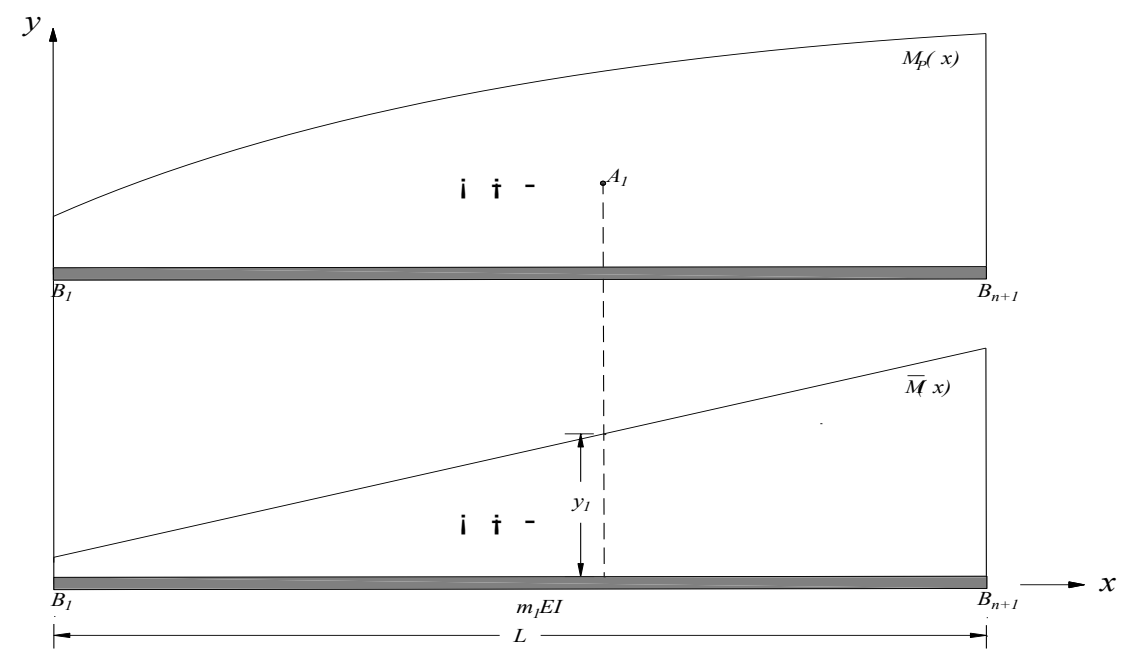

Figure 2. Graph multiplication of $\Delta_{l}$ in the $1^{s t}$ state. 


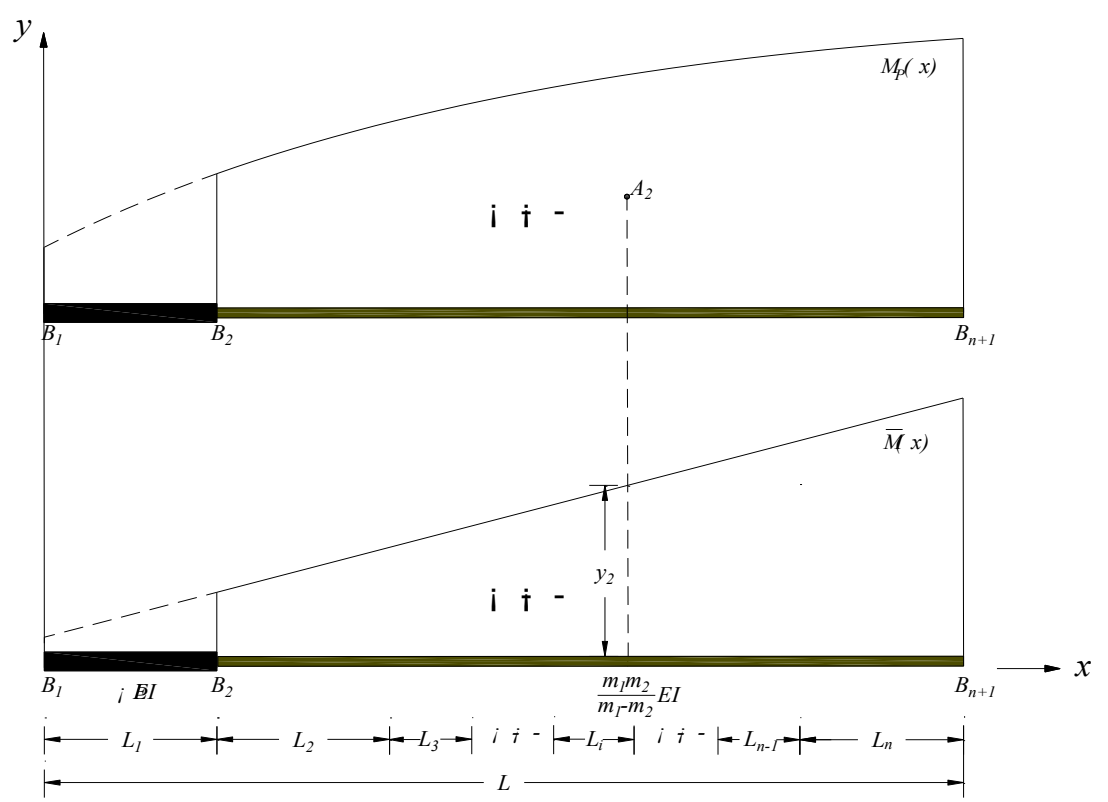

Figure 3. Graph multiplication of $\Delta_{2}$ in the $2^{\text {nd }}$ state.

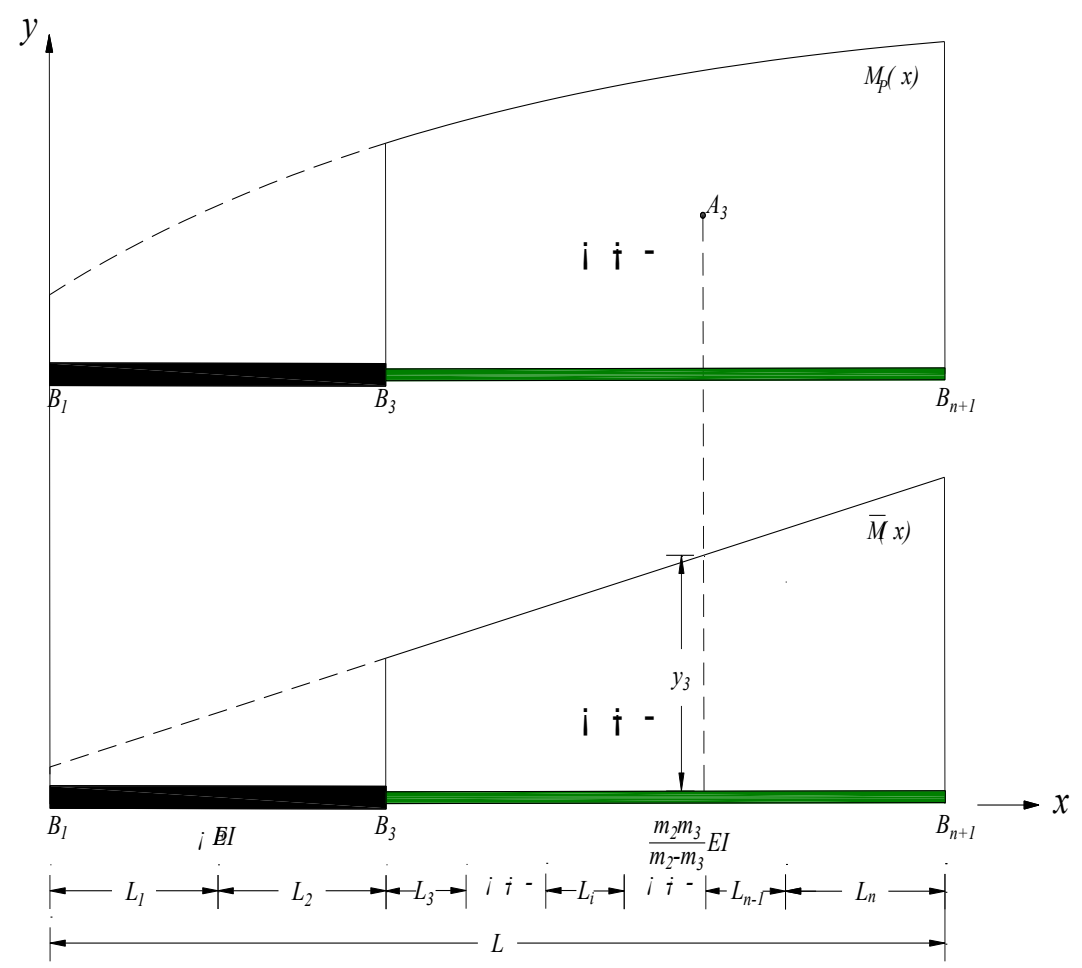

Figure 4. Graph multiplication of $\Delta_{3}$ in the $3^{r d}$ state. 


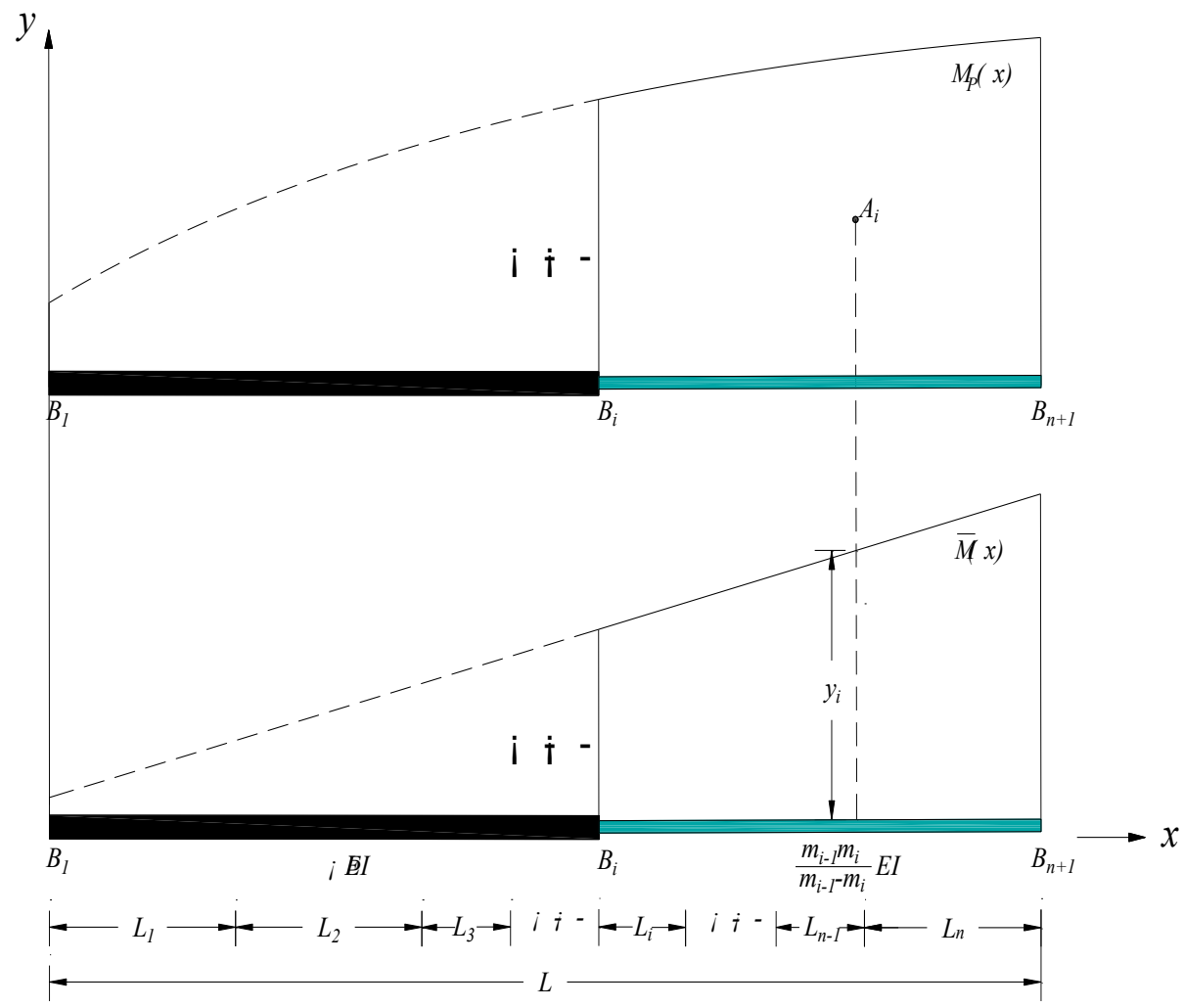

Figure 5. Graph multiplication of $\Delta_{i}$ in the $i^{\text {th }}$ state.

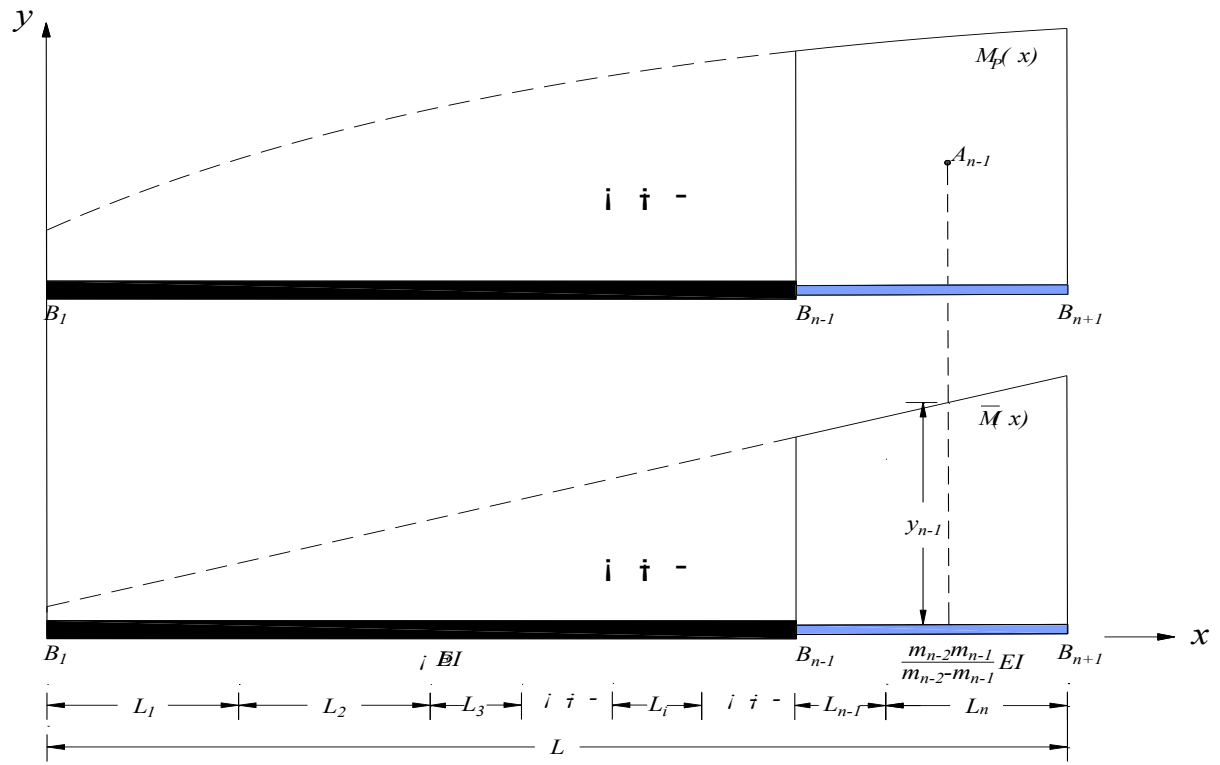

Figure 6. Graph multiplication of $\Delta_{n-1}$ in the $n-1^{\text {th }}$ state. 


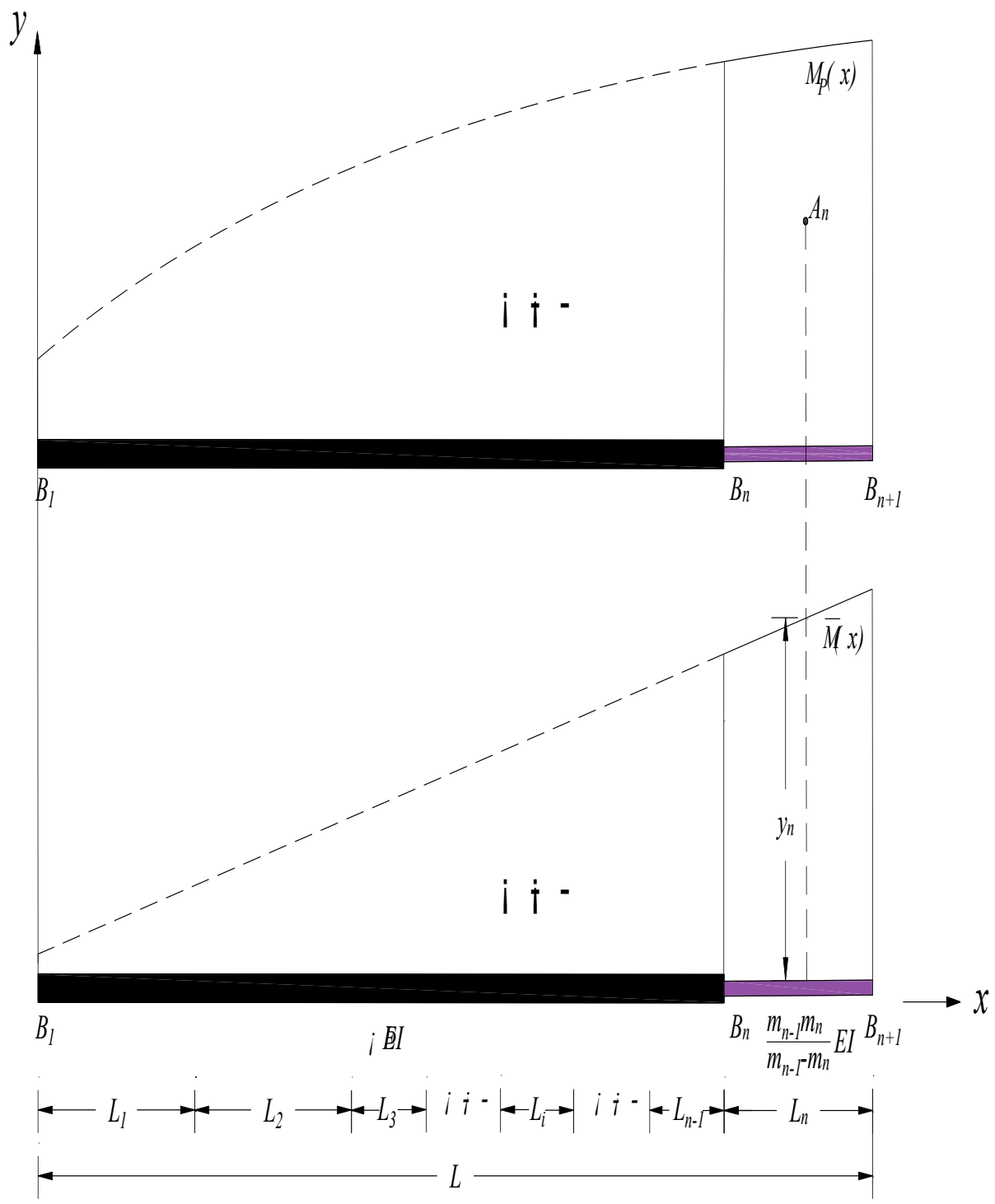

Figure 7. Graph multiplication of $\Delta_{n}$ in the $n^{\text {th }}$ state.

In the above six figures, $A_{1}, A_{2}, A_{3}, A_{i}, A_{n-1}$ and $A_{n}$ are the areas of the solid-line parts of the bending moment $M_{P}(x)$ diagrams, whereas $y_{1}, y_{2}, y_{3}, y_{i}, y_{n-1}$ and $y_{n}$ are the vertical coordinates of the $\bar{M}(x)$ diagrams corresponding to the centroids of the areas. According to the basic principle of the CGM, we obtain 


$$
\begin{aligned}
& \Delta_{1}=\frac{1}{m_{1} E I} \int_{0}^{L} \bar{M}(x) M_{P}(x) \mathrm{d} x=\frac{1}{m_{1} E I} A_{1} y_{1} \\
& \Delta_{2}=\frac{m_{1}-m_{2}}{m_{1} m_{2} E I} \int_{L_{1}}^{L} \bar{M}(x) M_{P}(x) \mathrm{d} x \\
& =\frac{1}{\infty E I} \int_{0}^{L_{1}} \bar{M}(x) M_{P}(x) \mathrm{d} x+\frac{m_{1}-m_{2}}{m_{1} m_{2} E I} \int_{L_{1}}^{L} \bar{M}(x) M_{P}(x) \mathrm{d} x=\frac{m_{1}-m_{2}}{m_{1} m_{2} E I} A_{2} y_{2} \\
& \Delta_{3}=\frac{m_{2}-m_{3}}{m_{2} m_{3} E I} \int_{L_{1}+L_{2}}^{L} \bar{M}(x) M_{P}(x) \mathrm{d} x \\
& =\frac{1}{\infty E I} \int_{0}^{L_{1}+L_{2}} \bar{M}(x) M_{P}(x) \mathrm{d} x+\frac{m_{2}-m_{3}}{m_{2} m_{3} E I} \int_{L_{1}+L_{2}}^{L} \bar{M}(x) M_{P}(x) \mathrm{d} x=\frac{m_{2}-m_{3}}{m_{2} m_{3} E I} A_{3} y_{3}
\end{aligned}
$$

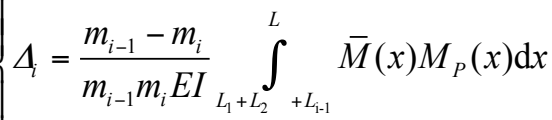

$$
\begin{aligned}
& =\frac{1}{\infty E I} \int_{0}^{L_{1}+L_{2}} \bar{M}(x) M_{P}(x) \mathrm{d} x+\frac{m_{i-1}-m_{i}}{m_{i-1} m_{i} E I} \int_{L_{1}+L_{2}}^{L} \bar{M}(x) M_{P}(x) \mathrm{d} x=\frac{m_{i-1}-m_{i}}{m_{i-1} m_{i} E I} A_{i} y_{i} \\
& \Delta_{n-1}=\frac{m_{n-2}-m_{n-1}}{m_{n-2} m_{n-1} E I} \int_{L_{1}+L_{2}}^{L} \bar{M}(x) M_{P}(x) \mathrm{d} x \\
& =\frac{1}{\infty E I} \int_{0}^{L_{1}+L_{2}}{ }^{+L_{n-2}} \bar{M}(x) M_{P}(x) \mathrm{d} x+\frac{m_{n-2}-m_{n-1}}{m_{n-2} m_{n-1} E I} \int_{L_{1}+L_{2}}^{L} \bar{M}(x) M_{P}(x) \mathrm{d} x=\frac{m_{n-2}-m_{n-1}}{m_{n-2} m_{n-1} E I} A_{n-1} y_{n-1} \\
& \Delta_{n}=\frac{m_{n-1}-m_{n}}{m_{n-1} m_{n} E I} \int_{L_{1}+L_{2}}^{L} \bar{M}(x) M_{P}(x) \mathrm{d} x
\end{aligned}
$$

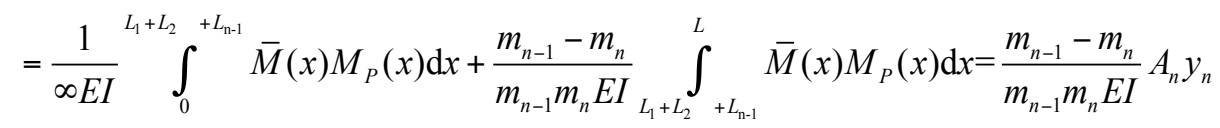

Then, we obtain

$$
\begin{aligned}
\Delta & =\sum_{i=1}^{n} \Delta_{i} \\
& =\frac{1}{m_{1} E I} A_{1} y_{1}+\sum_{i=2}^{n} \frac{m_{i-1}-m_{i}}{m_{i-1} m_{i} E I} A_{i} y_{i}
\end{aligned}
$$

The above derivation process shows that the core of the new method - the SDM — is the stiffness decomposition of the structure. For the SDM, Equation (9) is the general computation equation of displacement for a general nsegment complex bending structure. 


\section{Construction of Operational Principles}

To facilitate the application of the SDM for complex bending structures, its operational principles were established as follows:

(a) The rigidity of each segment of an $n$-segment complex bending structure is decomposed into $n$ parts in sequence. The reciprocal sum of the $n$-part postdecomposition stiffness of each segment is equal to the reciprocal of its original stiffness.

(b) The stiffness decomposition of the $1^{s t}$ stiffness decomposition state is carried out first. The stiffness of segment $\# 1$ of the structure is totally distributed to the $1^{\text {st }}$ postdecomposition stiffness along the total length of the structure. The postdecomposition stiffness of this segment in the subsequent states of stiffness decomposition is equal to $\infty E I$. After the stiffness decomposition of segment $\# 1$, the stiffnesses of the subsequent segments decrease along the length of the subsequent structure. The residual stiffness of segment $\# i$ of the structure is totally distributed to the $i^{\text {th }}$ postdecomposition stiffness along the subsequent structural length $L-\left(L_{1}+L_{2}+\ldots \ldots L_{i-1}\right)$. The postdecomposition stiffness of segment $\# i$ in the subsequent states of stiffness decomposition is $\infty E I$. The process does not stop until reaching the $n^{\text {th }}$ segment.

(c) The displacement of the structure in $n$ states of decomposition stiffness is calculated with the CGM, as shown in Equation (8). The displacements corresponding to all states are superposed to give the final undetermined displacement $\Delta$ of the structure, as shown in Equation (9).

Based on the above procedure, the operation flow of the SDM is illustrated in Table 2.

Table 2. Operation flow of the SDM.

\begin{tabular}{|c|c|c|c|c|c|c|c|c|c|}
\hline \multirow[b]{2}{*}{ Segment } & \multicolumn{8}{|c|}{ Stiffness corresponding to each state of stiffness decomposition } & \multirow{2}{*}{$\begin{array}{l}\begin{array}{l}\text { Stiffness in } \\
\text { initial state }\end{array} \\
\begin{array}{l}\text { Reciprocal of } \\
\text { initial stiffness } \\
\Sigma(i)\end{array} \\
\end{array}$} \\
\hline & $\begin{array}{l}\text { Reciprocal of the } 1^{\text {st }} \\
\text { postdecomposition } \\
\text { stiffness in the } 1^{\text {st }} \\
\text { state (1) }\end{array}$ & $\begin{array}{l}\text { Reciprocal of the } 2^{\text {nd }} \\
\text { postdecomposition } \\
\text { stiffness in the } 2^{\text {nd }} \\
\text { state (2) }\end{array}$ & $\begin{array}{l}\text { Reciprocal of the } 3^{\text {rd }} \\
\text { postdecomposition } \\
\text { stiffness in the } 3^{\text {rd }} \\
\text { state (3) }\end{array}$ & $\cdots \cdots$ & $\begin{array}{l}\text { Reciprocal of the } i^{\text {th }} \\
\text { postdecomposition } \\
\text { stiffness in the } i^{\text {th }} \\
\text { state (i) }\end{array}$ & $\ldots \ldots$ & $\begin{array}{l}\text { Reciprocal of the } n-I^{\text {th }} \\
\text { postdecomposition } \\
\text { stiffness in the } n-1^{\text {th }} \\
\text { state }(n-1)\end{array}$ & $\begin{array}{l}\text { Reciprocal of the } n^{\text {th }} \\
\text { postdecomposition } \\
\text { stiffness in the } n^{\text {th }} \\
\text { state (n) }\end{array}$ & \\
\hline$\# 1$ & $1 / m_{1} E I$ & $1 / \infty E I$ & $1 / \infty E I$ & $\ldots \ldots$ & $1 / \infty E I$ & $\ldots \ldots$ & $1 / \infty E I$ & $1 / \infty E I$ & $1 / m_{1} E I$ \\
\hline \#2 & $1 / m_{l} E I$ & $\left(m_{1}-m_{2}\right) / m_{1} m_{2} E I$ & $1 / \infty E I$ & $\ldots \ldots$ & $1 / \infty E I$ & $\ldots \ldots$ & $1 / \infty E I$ & $1 / \infty E I$ & $1 / m_{2} E I$ \\
\hline \#3 & $1 / m_{l} E I$ & $\left(m_{1}-m_{2}\right) / m_{l} m_{2} E I$ & $\left(m_{2}-m_{3}\right) / m_{2} m_{3} E I$ & $\ldots . .$. & $1 / \infty E I$ & $\ldots \ldots$ & $1 / \infty E I$ & $1 / \infty E I$ & $1 / m_{3} E I$ \\
\hline$\ldots \ldots$ & ....... & $\ldots \ldots$ & $\ldots \ldots$ & $\ldots \ldots$ & $\ldots \ldots$ & $\ldots \ldots$ & $\cdots \cdots$ & $\ldots \ldots$ & $\ldots \ldots$ \\
\hline$\# i$ & $1 / m_{l} E I$ & $\left(m_{1}-m_{2}\right) / m_{l} m_{2} E I$ & $\left(m_{2}-m_{3}\right) / m_{2} m_{3} E I$ & $\ldots \ldots$ & $\left(m_{i-1}-m_{i}\right) / m_{i-l} m_{i} E I$ & $\ldots \ldots$ & $1 / \infty E I$ & $1 / \infty E I$ & $I / m_{i} E I$ \\
\hline$\ldots \ldots$ & $\ldots \ldots$ & $\ldots \ldots$ & $\ldots \ldots$ & $\ldots \ldots$ & $\ldots \ldots$ & $\ldots \ldots$ & $\ldots \ldots$ & $\ldots \ldots$ & $\ldots \ldots$ \\
\hline$\# n-1$ & $1 / m_{l} E I$ & $\left(m_{1}-m_{2}\right) / m_{1} m_{2} E I$ & $\left(m_{2}-m_{3}\right) / m_{2} m_{3} E I$ & $\ldots \ldots$ & $\left(m_{i-1}-m_{i}\right) / m_{i-l} m_{i} E I$ & $\ldots \ldots$ & $\left(m_{n-2}-m_{n-l}\right) / m_{n-2} m_{n-l} E I$ & $1 / \infty E I$ & $1 / m_{n-1} E I$ \\
\hline$\# n$ & $1 / m_{l} E I$ & $\left(m_{1}-m_{2}\right) / m_{1} m_{2} E I$ & $\left(m_{2}-m_{3}\right) / m_{2} m_{3} E I$ & $\ldots \ldots$ & $\left(m_{i-1}-m_{i}\right) / m_{i-1} m_{i} E I$ & $\ldots$ & $\left(m_{n-2}-m_{n-1}\right) / m_{n-2} m_{n-1} E I$ & $\left(m_{n-l}-m_{n}\right) / m_{n-l} m_{n} E I$ & $1 / m_{n} E I$ \\
\hline $\begin{array}{c}\text { Graph } \\
\text { multiplication }\end{array}$ & Fig. 2 & Fig. 3 & Fig. 4 & $\ldots \ldots$ & Fig. 5 & $\ldots \ldots$ & Fig. 6 & Fig. 7 & l \\
\hline Displacement & $\Delta_{l}($ Eq. $(8))$ & $\Delta_{2}$ (Eq. (8)) & $\Delta_{3}($ Eq. $(8))$ & $\ldots \ldots$ & $\Delta_{i}$ (Eq. (8)) & $\ldots \ldots$ & $\Delta_{n-l}$ (Eq. (8)) & $\Delta_{n}($ Eq. (8)) & $\Delta=\sum_{i=1}^{n} \Delta_{i}$ (Eq. (9)) \\
\hline
\end{tabular}

All of the components of the desired displacement in Equation (8) and Equation (9), namely, $\Delta_{l}, \Delta_{2} \ldots \ldots \Delta_{n}$, correspond to each state of stiffness decomposition. 


\section{CASE STUDY: COMPLEX CANTILEVER BEAM}

The complex cantilever beam structure shown in Figure 8 was taken as the first example to show the application of the SDM. This structure has three segments. The structure is fixed at point $B_{l}$. The vertical displacement $\Delta_{B_{4}}$ at the cantilever end, point $B_{4}$, needs to be solved under uniform load $q$. Based on the basic principle of the unit load method, a vertical unit load $P=1$ is exerted at point $B_{4}$ along the vertical direction, as shown in Figure 8 .

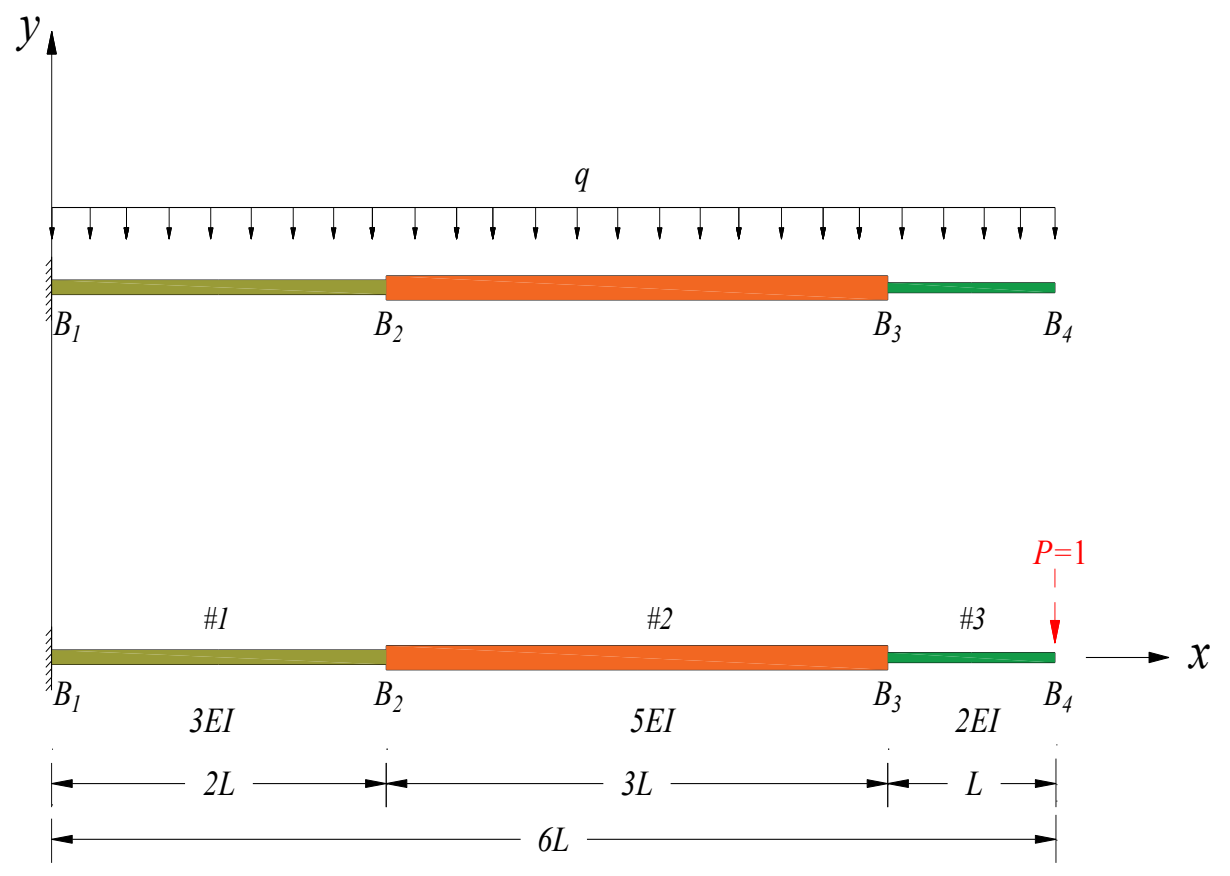

Figure 8. Complex cantilever beam.

\section{Direct Integration Method}

First, the DIM was implemented to calculate the displacement of the structure shown in Figure 8. According to the unit load method, the bending moment functions $\bar{M}(x)$ and $M_{P}(x)$ of the structure can be easily determined by simple internal force analyses. The results are shown in

$$
\begin{cases}\bar{M}(x)=6 L-x & (0<x \leq 6 L) \\ M_{P}(x)=\frac{1}{2} q(6 L-x)^{2} & (0 \leq x \leq 6 L)\end{cases}
$$

Based on Equation (1) and considering that $d_{s}=d_{x}$ for straight bars, the calculation process and result of the displacement are expressed in 


$$
\begin{aligned}
\Delta_{B_{4}} & =\sum \int_{0}^{L} \frac{\bar{M}(x) M_{P}(x)}{E I(x)} \mathrm{d} x \\
& =\frac{1}{3 E I} \int_{0}^{2 L} \frac{1}{2} q(6 L-x)^{3} \mathrm{~d} x+\frac{1}{5 E I} \int_{2 L}^{5 L} \frac{1}{2} q(6 L-x)^{3} \mathrm{~d} x+\frac{1}{2 E I} \int_{5 L}^{6 L} \frac{1}{2} q(6 L-x)^{3} \mathrm{~d} x \\
& =\frac{q}{6 E I} \int_{0}^{2 L}\left(216 L^{3}-108 L^{2} x+18 L x^{2}-x^{3}\right) \mathrm{d} x \\
& +\frac{q}{10 E I} \int_{2 L}^{5 L}\left(216 L^{3}-108 L^{2} x+18 L x^{2}-x^{3}\right) \mathrm{d} x+\frac{q}{4 E I} \int_{5 L}^{6 L}\left(216 L^{3}-108 L^{2} x+18 L x^{2}-x^{3}\right) \mathrm{d} x \\
& =\left.\frac{q}{6 E I}\left(216 L^{3} x-54 L^{2} x^{2}+6 L x^{3}-\frac{1}{4} x^{4}\right)\right|_{0} ^{2 L} \\
& +\left.\frac{q}{10 E I}\left(216 L^{3} x-54 L^{2} x^{2}+6 L x^{3}-\frac{1}{4} x^{4}\right)\right|_{2 L} ^{5 L}+\left.\frac{q}{4 E I}\left(216 L^{3} x-54 L^{2} x^{2}+6 L x^{3}-\frac{1}{4} x^{4}\right)\right|_{5 L} ^{6 L} \\
& =\frac{q L^{4}}{6 E I}\left(216 \times 2-54 \times 2^{2}+6 \times 2^{3}-\frac{1}{4} \times 2^{4}\right) \\
& +\frac{q L^{4}}{10 E I}\left[\left(216 \times 5-54 \times 5^{2}+6 \times 5^{3}-\frac{1}{4} \times 5^{4}\right)-\left(216 \times 2-54 \times 2^{2}+6 \times 2^{3}-\frac{1}{4} \times 2^{4}\right)\right] \\
& +\frac{q L^{4}}{4 E I}\left[\left(216 \times 6-54 \times 6^{2}+6 \times 6^{3}-\frac{1}{4} \times 6^{4}\right)-\left(216 \times 5-54 \times 5^{2}+6 \times 5^{3}-\frac{1}{4} \times 5^{4}\right)\right] \\
& =\left(\frac{130}{3}+\frac{51}{8}+\frac{1}{16}\right) \frac{q L^{4}}{E I} \\
& =\frac{2389 q L^{4}}{48 E I}
\end{aligned}
$$

Equation (11) shows that the DIM requires three single integral operations for the displacement calculation. Each single integral operation requires at least five steps: factor substitution, factor product, indefinite integration, definite integration and factor addition. The calculation of each step in this operation is very complex and inconvenient.

\section{Conventional Graph Multiplication Method}

If the displacement of the structure shown in Figure 8 is computed by the CGM, the bending moment $\bar{M}(x)$ and $M_{P}(x)$ diagrams under the unit load and the actual uniform load should be determined first. These diagrams are illustrated in Figure 9 according to the basic principle of this method. 


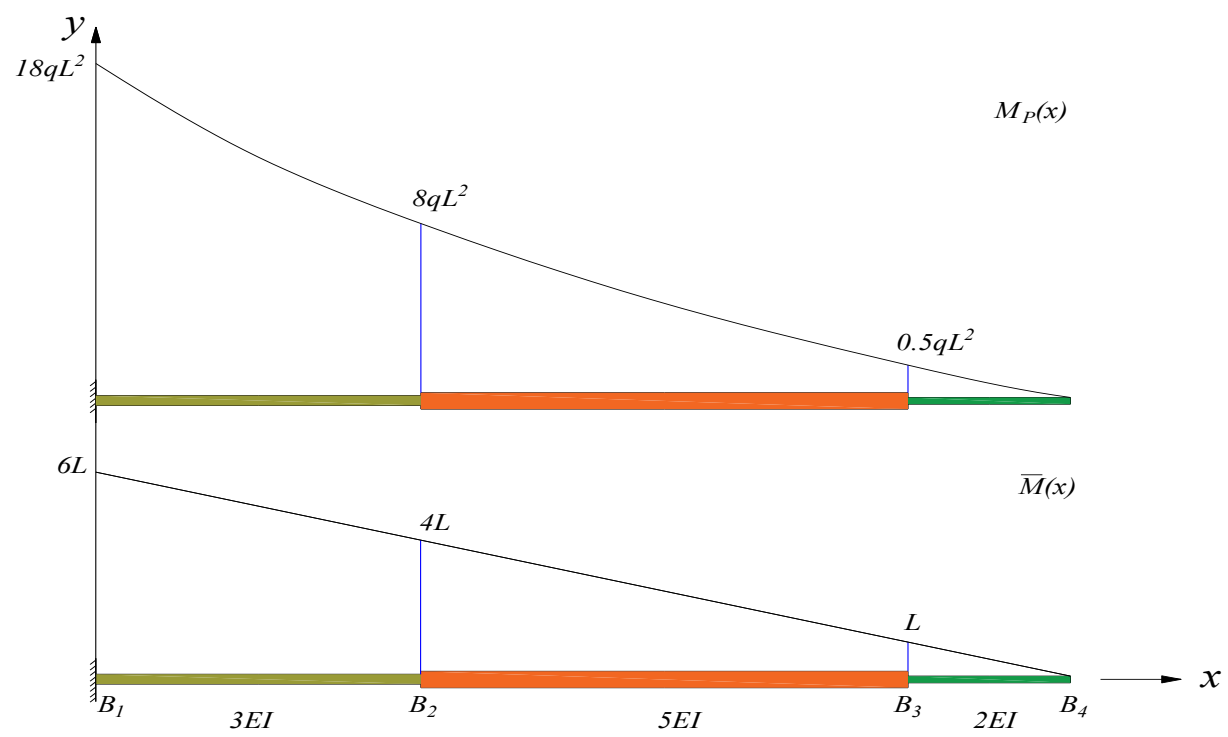

Figure 9. $\bar{M}(x)$ and $M_{P}(x)$ diagrams of the cantilever beam under $P=1$ and actual loads.

Figure 9 shows that the bending moment $M_{P}(x)$ diagrams belonging to each segment are not all standard diagrams. Therefore, the segments must be subdivided into blocks to apply the CGM to calculate the displacement. The simplest subdivision is shown in Figure 10 by referring to the standard diagrams in Table 1.

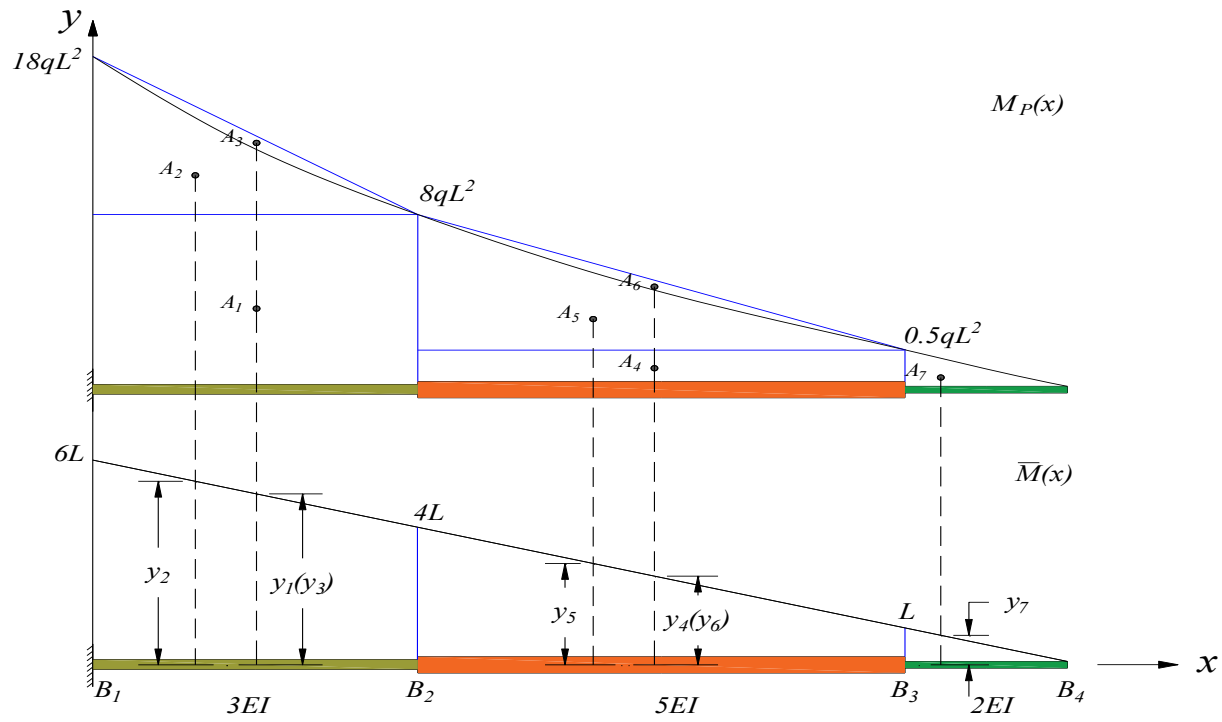

Figure 10. Conventional graph multiplication of the cantilever beam.

Through a comparison with the standard diagrams in Table 1, the areas of the blocks were determined, as shown in Equation (12). The vertical ordinates of the linear bending moment $\bar{M}(x)$ diagrams corresponding to the centroids of each area block were also computed, and the results are shown in 


$$
\left\{\begin{array}{l}
A_{1}=16 q L^{3}, \quad y_{1}=5 L ; \quad A_{2}=10 q L^{3}, \quad y_{2}=\frac{16 L}{3} ; \quad A_{3}=\frac{2 q L^{3}}{3}, y_{3}=y_{1} ; \\
A_{4}=\frac{3 q L^{3}}{2}, \quad y_{4}=\frac{5 L}{2} ; \quad A_{5}=\frac{45 q L^{3}}{4}, y_{5}=3 L ; \quad A_{6}=\frac{9 q L^{3}}{4}, y_{6}=y_{4} ; A_{7}=\frac{q L^{3}}{6}, y_{7}=\frac{3 L}{4}
\end{array}\right.
$$

Based on the CGM, the displacement calculation process and result of the cantilever beam by using Equation (2) are shown in

$$
\begin{aligned}
D_{B_{4}} & =\hat{\mathrm{A}} \frac{A_{i} y_{i}}{(E I)_{i}}=\frac{1}{3 E I}\left(A_{1} y_{1}+A_{2} y_{2}-A_{3} y_{3}\right)+\frac{1}{5 E I}\left(A_{4} y_{4}+A_{5} y_{5}-A_{6} y_{6}\right)+\frac{1}{2 E I} A_{7} y_{7} \\
& =\frac{2389 q L^{4}}{48 E I}
\end{aligned}
$$

A comparison between Equation (13) and Equation (11) shows that the CGM is slightly simpler than the DIM. However, the area $A_{i}$ and the graph multiplications require seven computations, and there are five computations for the vertical ordinate $y_{i}$. Therefore, the CGM still requires a high number of operations, which makes the calculation process tedious and complex.

\section{Stiffness Decomposition Method}

If the SDM is applied to calculate the displacement of the beam in Figure 8, we should compare the case with the general $n$-segment complex bending structure shown in Figure 1. It is obvious that $m_{1}=3, m_{2}=5$, and $m_{3}=2$ based on the basic principle of the method. According to the operational principles proposed in Table 2, the stiffness

\begin{tabular}{|c|c|c|c|c|}
\hline \multirow[b]{2}{*}{ Segment } & \multicolumn{3}{|c|}{ Stiffness corresponding to each state of stiffness decomposition } & $\begin{array}{c}\text { Stiffness in initial } \\
\text { state }\end{array}$ \\
\hline & $\begin{array}{l}\text { Reciprocal of the } 1^{\text {st }} \\
\text { postdecomposition } \\
\text { stiffness in the } 1^{\text {st }} \text { state } \\
\text { (1) }\end{array}$ & $\begin{array}{l}\text { Reciprocal of the } 2^{\text {nd }} \\
\text { postdecomposition } \\
\text { stiffness in the } 2^{\text {nd }} \\
\text { state (2) }\end{array}$ & $\begin{array}{l}\text { Reciprocal of the } 3^{\text {rd }} \\
\text { postdecomposition } \\
\text { stiffness in the } 3^{\text {rd }} \\
\text { state (3) }\end{array}$ & $\begin{array}{l}\text { Reciprocal of } \\
\text { initial stiffness } \\
\text { (1) }+ \text { (2) }+ \text { (3) }\end{array}$ \\
\hline$\# 1$ & $1 / 3 E I$ & $1 / \infty E I$ & $1 / \infty E I$ & $1 / 3 E I$ \\
\hline$\# 2$ & $1 / 3 E I$ & $-1 / 7.5 E I$ & $1 / \infty E I$ & $1 / 5 E I$ \\
\hline
\end{tabular}
decomposition flow of the structure was constructed as shown in Table 3.

Table 3. Stiffness decomposition of the cantilever beam. 


\begin{tabular}{|c|c|c|c|c|}
\hline$\# 3$ & $1 / 3 E I$ & $-1 / 7.5 E I$ & $3 / 10 E I$ & $1 / 2 E I$ \\
\hline $\begin{array}{c}\text { Graph } \\
\text { multiplication }\end{array}$ & Figure 11 (a) & Figure 11 (b) & Figure 11 (c) & $/$ \\
\hline Displacement & $\Delta_{1}$ (Equation (8)) & $\Delta_{2}$ (Equation (8)) & $\Delta_{3}$ (Equation (8)) & $\begin{array}{c}\Delta_{=}+\Delta_{1}+\Delta_{2}+\Delta_{3} \\
\text { (Equation }(9))\end{array}$ \\
\hline
\end{tabular}

After the stiffness decomposition, graph multiplication was conducted for each state of the stiffness decomposition, as shown in Figure 11 based on Table 3.

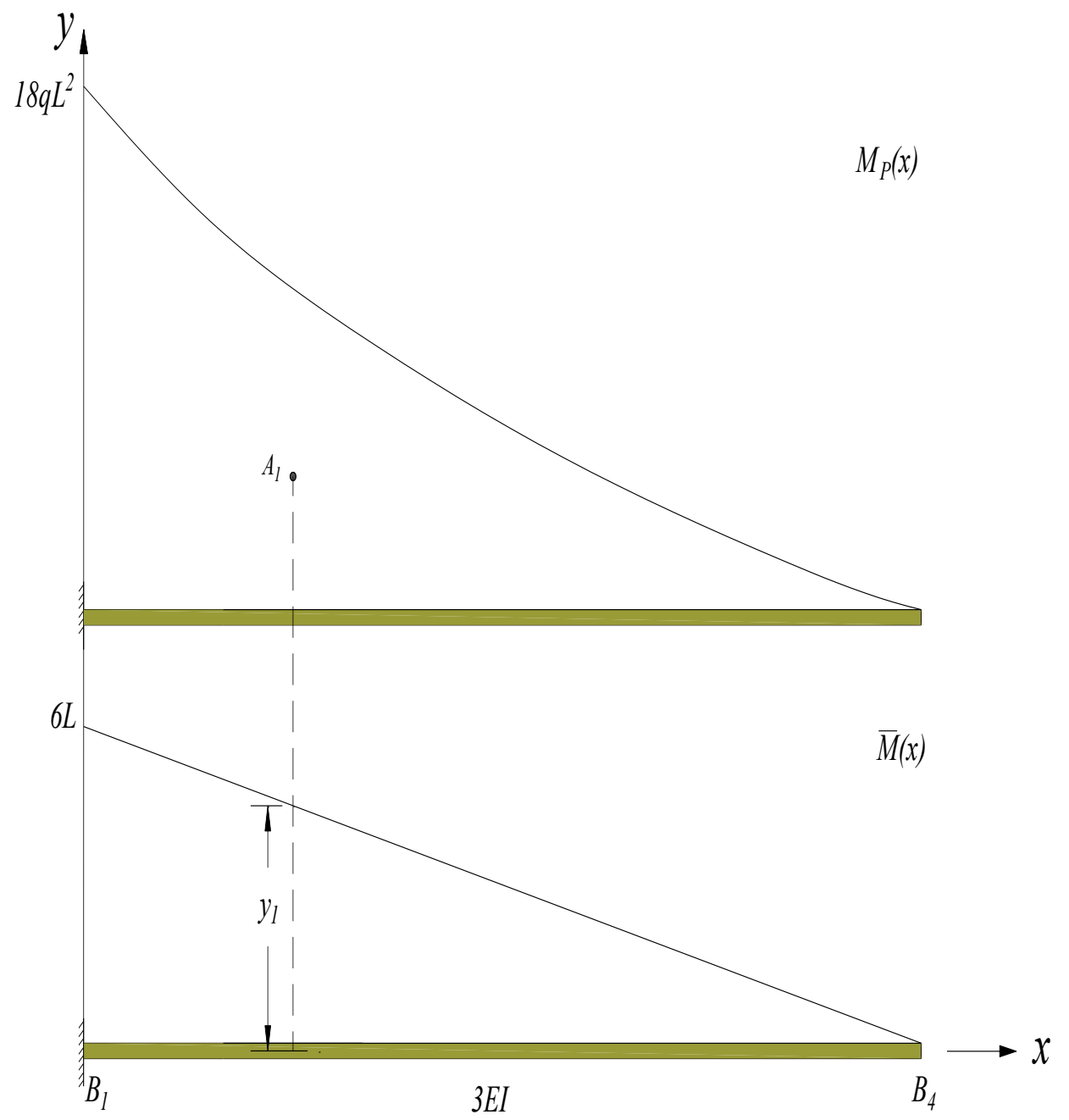

(a) $\Delta_{l}$ of the $1^{\text {st }}$ state 


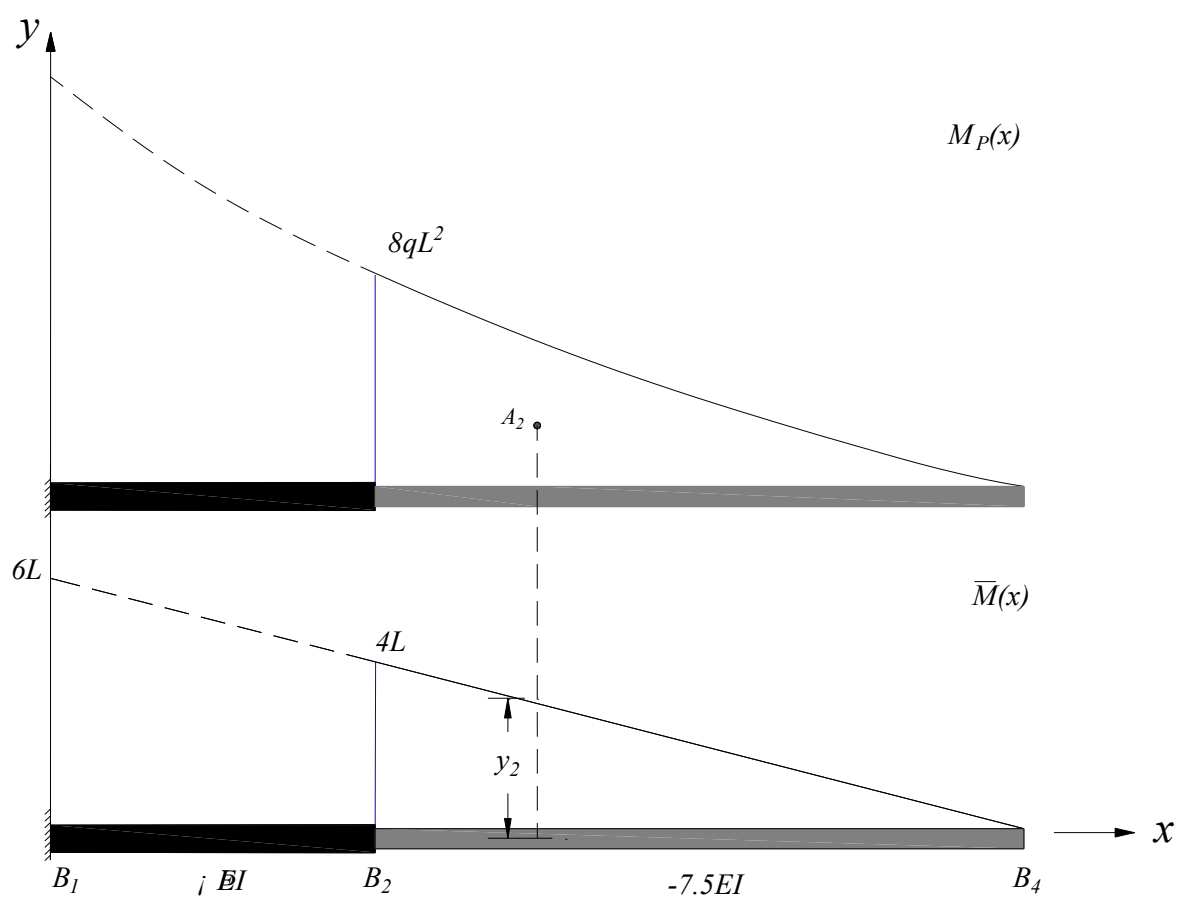

(b) $\Delta_{2}$ of the $2^{\text {nd }}$ state

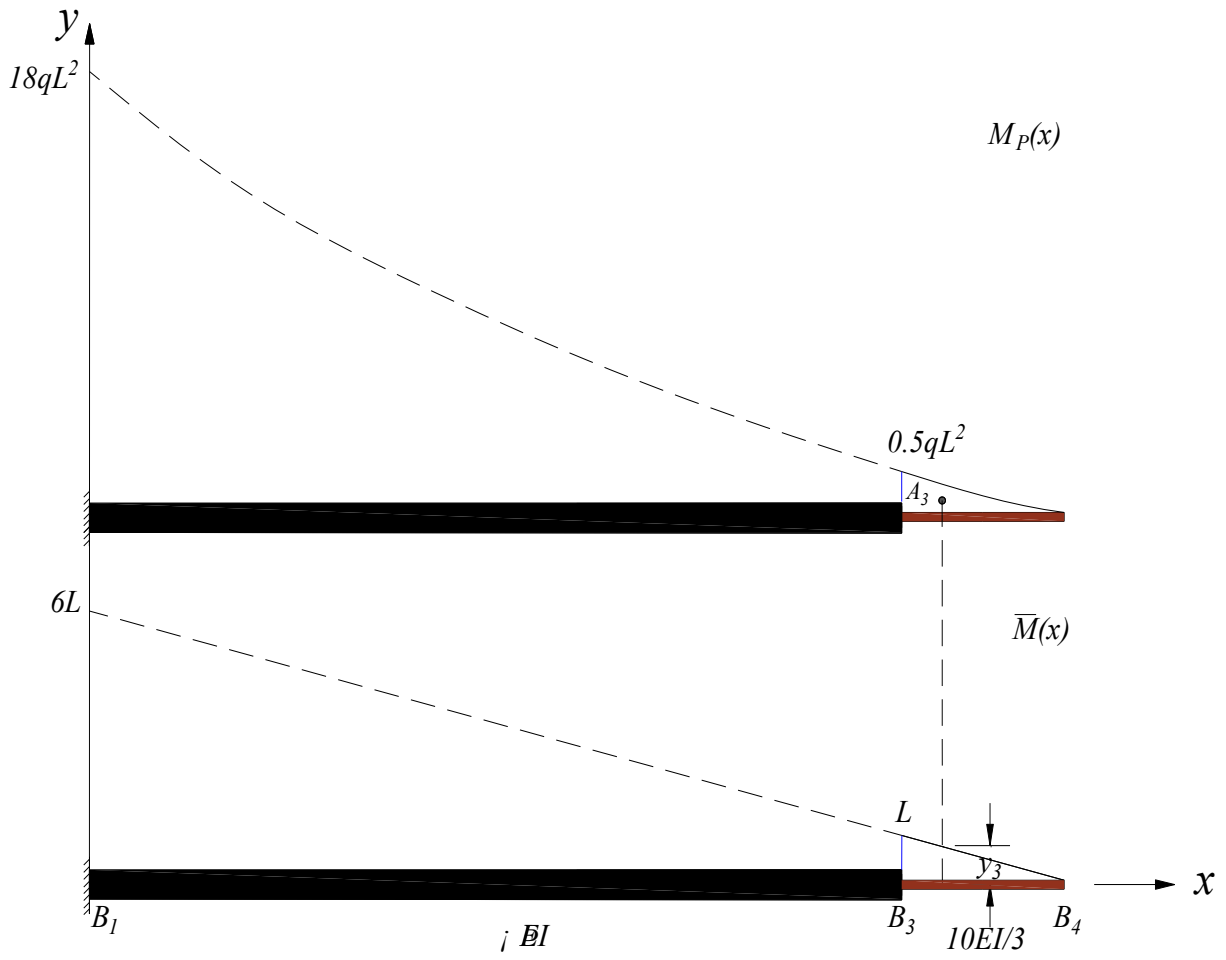


(c) $\Delta_{3}$ of the $3^{r d}$ state

Figure 11. Graph multiplication of each state for the cantilever beam via the SDM.

Because the corresponding stiffness is $\infty E I$, the calculated displacement is zero for the graph multiplication of the dashed bending moment diagrams in Figure 11. Therefore, only graph multiplications of the solid bending moment diagrams are required. A comparison with Table 1 shows that the solid bending moment $M_{P}(x)$ diagrams in Figure 11 are all standard diagrams. Therefore, the areas and centroids of these diagrams can be easily determined. The corresponding vertical coordinates of the linear bending moment $\bar{M}(x)$ diagrams were also computed. The results are shown in Equation (14).

$$
A_{1}=36 q L^{3}, \quad y_{1}=\frac{9 L}{2} ; \quad A_{2}=\frac{32 q L^{3}}{3}, y_{2}=3 L ; \quad A_{3}=\frac{q L^{3}}{6}, y_{3}=\frac{3 L}{4}
$$

Then, Equation (9) was applied to compute the displacement of the cantilever beam, as shown in

$$
D_{B_{4}}=\hat{\AA}_{i=1}^{3} D_{i}=\frac{1}{m_{1} E I} A_{1} y_{1}+\underset{i=2}{\hat{A}_{1-1}^{3}} \frac{m_{i-1}-m_{i}}{m_{i-1} m_{i} E I} A_{i} y_{i}=\frac{1}{3 E I} A_{1} y_{1}-\frac{1}{7.5 E I} A_{2} y_{2}+\frac{3}{10 E I} A_{3} y_{3}=\frac{2389 q L^{4}}{48 E I}
$$

\section{Comparison and Discussion}

A comparison between Equation (15) and Equation (11) shows that the displacement calculated by the SDM is the same as that calculated by the DIM. From the perspective of the total number of mathematical operations, a comparison between the two methods is shown in Table 4 . 
Table 4. Comparison between the SDM and DIM for the cantilever beam.

\begin{tabular}{|c|c|c|c|c|c|}
\hline Method & \multicolumn{4}{|c|}{ Number of mathematical operations } & \multirow{2}{*}{$\begin{array}{c}\text { Complexity } \\
\text { Each operation }\end{array}$} \\
\hline \multirow{2}{*}{ SDM (1) } & $A_{i}$ (3) & $y_{i}$ (4) & $\begin{array}{c}\text { Graph multiplication } \\
\text { (5) }\end{array}$ & $\begin{array}{c}\text { Total } \\
(3)+(4)+(5)\end{array}$ & \\
\hline & 3 & 3 & 3 & 9 & Simple \\
\hline \multirow{2}{*}{ DIM (2) } & $\begin{array}{c}\text { Single } \\
\text { integrations } \\
\text { 6) }\end{array}$ & $\begin{array}{l}\text { Operation steps } \\
\text { per integration } \\
(7)\end{array}$ & / & $\begin{array}{l}\text { Total } \\
\text { (6) } \times(7)\end{array}$ & Each operation \\
\hline & 3 & 5 & / & 15 & Complicated \\
\hline (1)/(2) & / & I & 1 & $60.0 \%$ & 1 \\
\hline
\end{tabular}

Table 4 shows that the total operation numbers of the SDM account for $60.0 \%$ of those in the DIM. Each operation in the SDM is very simple, whereas each operation in the DIM is very complex.

If Equation (15) is compared with Equation (13), the displacement result of the SDM is also equal to that of the CGM. Considering the complexity of graph multiplication, a comparison between the SDM and CGM is illustrated in Table 5.

Table 5. Comparison between the SDM and CGM for the cantilever beam.

\begin{tabular}{|c|c|c|c|c|}
\hline \multirow{2}{*}{ Method } & \multicolumn{5}{|c|}{ Number of mathematical operations } \\
\cline { 2 - 5 } & $\mathbf{A}_{\mathbf{i}}(3)$ & $\mathbf{y}_{\mathbf{i}}(4)$ & Graph multiplication (5) & Total (3)+(4)+(5) \\
\hline SDM (1) & 3 & 3 & 3 & 9 \\
\hline CGM (2) & 7 & 5 & 7 & 19 \\
\hline (1)/(2) & $42.9 \%$ & $60.0 \%$ & $42.9 \%$ & $47.4 \%$ \\
\hline
\end{tabular}


Table 5 shows that the SDM only accounts for $47.4 \%$ of the CGM in terms of the total number of mathematical operations. It can be estimated that these proportions will further decrease as the number of structural segments increases.

Therefore, the SDM is simpler and more efficient than the DIM and the CGM from comparisons of the three methods in the displacement calculation of the complex cantilever beam.

\section{CASE STUDY: COMPLEX SIMPLY SUPPORTED BEAM}

The complex simply supported beam illustrated in Figure 12 was taken as a second typical case to illustrate the application of the SDM. This structure is subjected to a vertical uniform load $q$. We assume that the vertical displacement of the midspan $O$ point of the structure must be solved. Based on the unit load method, the unit virtual load $P=1$ should be exerted at this point along the vertical direction, as shown in Figure 12.

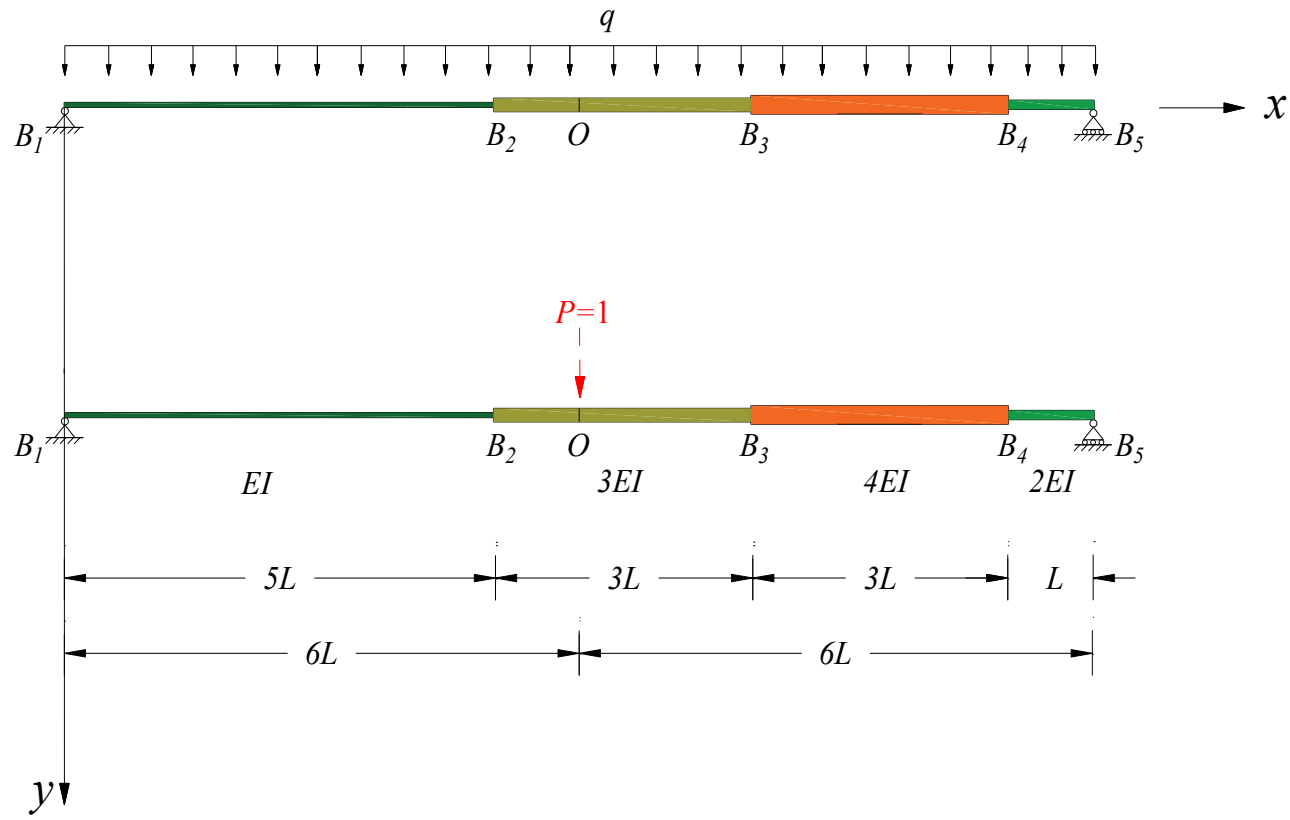

Figure 12. Complex simply supported beam.

\section{Direct Integration Method}

To calculate the displacement of the structure in Figure 12 with the DIM, the bending moment $\bar{M}(x)$ and $M_{P}(x)$ functions of the structure were calculated by simple internal force analyses; these functions correspond to the unit load $\mathrm{P}=1$ and the actual load, respectively. Both functions are shown in

$$
\bar{M}(x)= \begin{cases}\frac{1}{2} x & (0 x \leq 6 L) \\ \frac{1}{2}(12 L-x) & (6 L<x \leq 12 L)\end{cases}
$$




$$
M_{P}(x)=6 q L x-\frac{1}{2} q x^{2} \quad(0 \leq x \leq 12 L)
$$

Considering that $d_{s}=d_{x}$ for straight framed structures, the vertical displacement of the beam was computed with the DIM based on Equation (1). The calculation result of the displacement is shown in

$$
\begin{aligned}
\Delta_{O} & =\sum \int_{0}^{L} \frac{\bar{M}(x) M_{P}(x)}{E I(x)} \mathrm{d} x \\
& =\frac{1}{E I} \int_{0}^{5 L} \frac{1}{2} x\left(6 q L x-\frac{1}{2} q x^{2}\right) \mathrm{d} x+\frac{1}{3 E I} \int_{5 L}^{6 L} \frac{1}{2} x\left(6 q L x-\frac{1}{2} q x^{2}\right) \mathrm{d} x+\frac{1}{3 E I} \int_{6 L}^{8 L} \frac{1}{2}(12 L-x)\left(6 q L x-\frac{1}{2} q x^{2}\right) \mathrm{d} x \\
& +\frac{1}{4 E I} \int_{8 L}^{11 L} \frac{1}{2}(12 L-x)\left(6 q L x-\frac{1}{2} q x^{2}\right) \mathrm{d} x+\frac{1}{2 E I} \int_{11 L}^{12 L} \frac{1}{2}(12 L-x)\left(6 q L x-\frac{1}{2} q x^{2}\right) \mathrm{d} x \\
& =\frac{27557 q L^{4}}{192 E I}
\end{aligned}
$$

Equation (18) shows that the application of the DIM requires five single integral operations. Each operation also requires at least five steps: factor substitution, factor product, indefinite integration, definite integration and factor addition. Additionally, the operations in each step are cumbersome and inefficient, which is similar to the first case.

\section{Conventional Graph Multiplication Method}

If the CGM is applied to calculate the displacement of the complex simply supported beam, the bending moment $\bar{M}(x)$ and $M_{P}(x)$ diagrams under the unit load $P=1$ and the actual load should be determined first. These diagrams are illustrated in Figure 13.

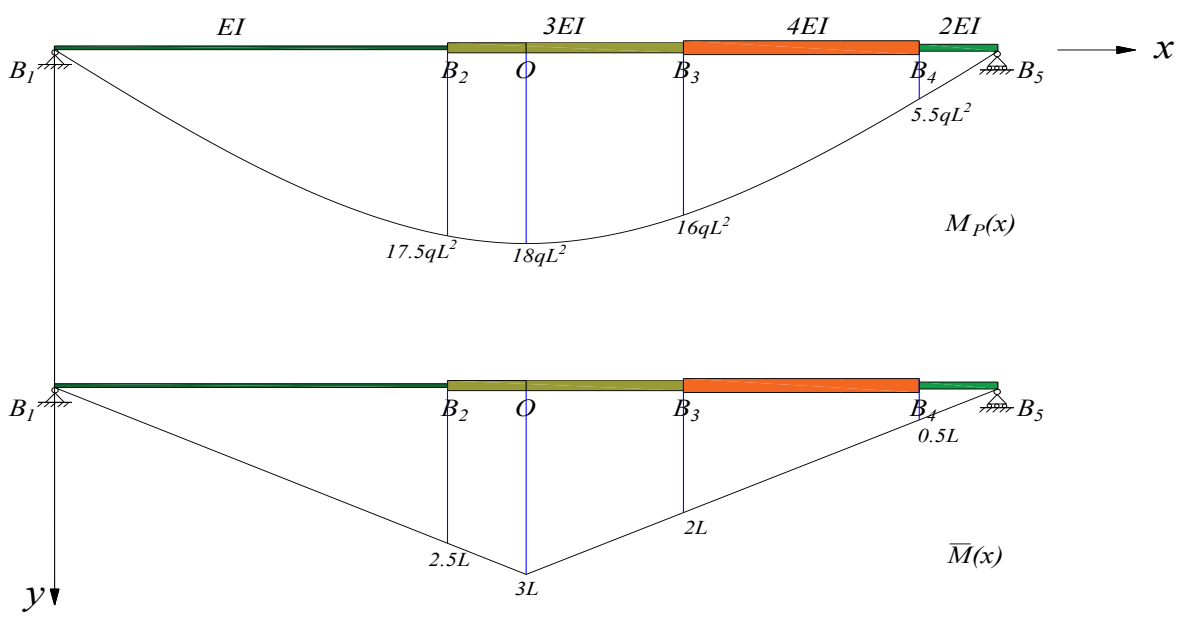

Figure 13. $\bar{M}(x)$ and $M_{P}(x)$ of the simply supported beam under $P=1$ and actual loads. 
There are two kinds of segments in Figure 13, the stiffness segment and the segment for the linear bending moment $\bar{M}(x)$ diagram. Therefore, the bending moment $M_{P}(x)$ diagram must be subdivided into blocks to use the standard diagrams in Table 1. The simplest segment and block subdivision are shown in Figure 14.

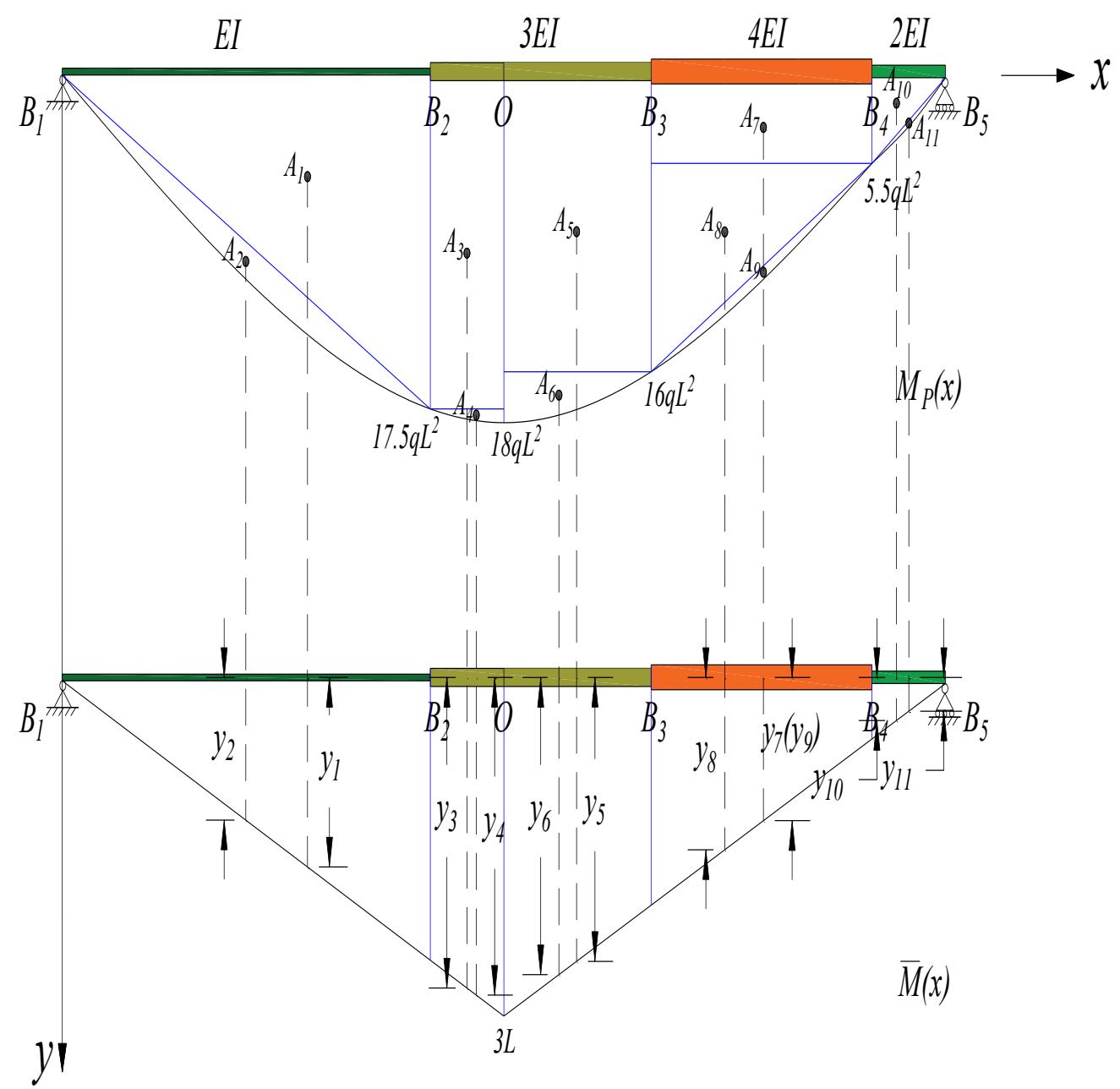

Figure 14. Conventional graph multiplication of the simply supported beam.

By comparing the blocks of the $M_{P}(x)$ diagram in Figure 14 with the standard diagrams in Table 1, the areas of the blocks and the vertical coordinates of the $\bar{M}(x)$ diagrams corresponding to the centroids of the area blocks were computed, and the results are shown in 


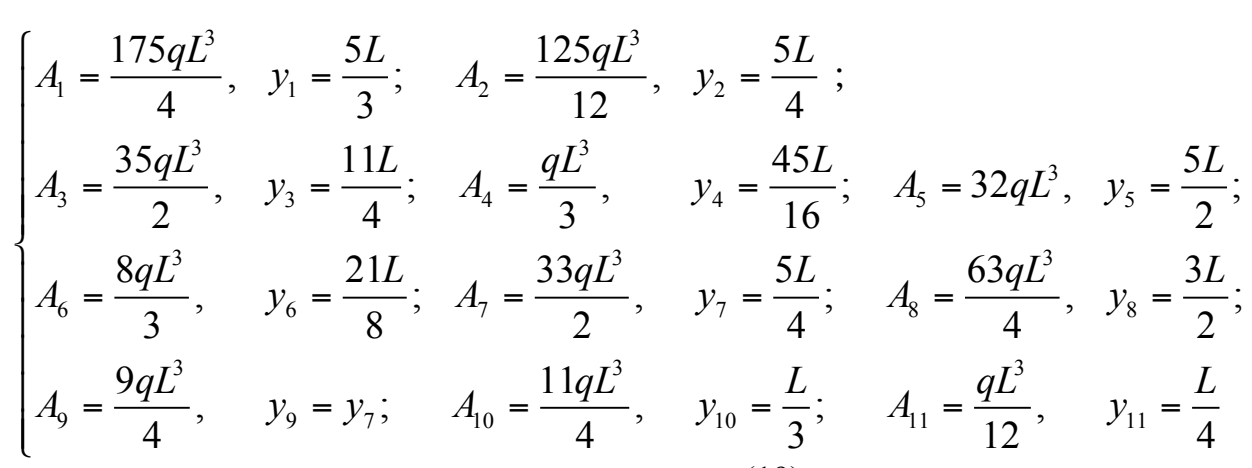

Based on the basic principle of the CGM, the displacement calculation process and results for the complex simply supported beam are shown in

$$
\begin{aligned}
D_{O} & =\hat{\mathrm{A}} \frac{A_{i} y_{i}}{(E I)_{i}} \\
& =\frac{1}{E I}\left(A_{1} y_{1}+A_{2} y_{2}\right)+\frac{1}{3 E I}\left(A_{3} y_{3}+A_{4} y_{4}+A_{5} y_{5}+A_{6} y_{6}\right)+\frac{1}{4 E I}\left(A_{7} y_{7}+A_{8} y_{8}+A_{9} y_{9}\right)+\frac{1}{2 E I}\left(A_{10} y_{10}+A_{11} y_{11}\right) \\
& =\frac{27557 q L^{4}}{192 E I}
\end{aligned}
$$

The results show that the CGM is slightly simpler than the DIM for the displacement calculation of the simply supported beam. However, the number of calculations for $A_{i}$ and the number of graph multiplications are all eleven, as shown in Equation (19) and Equation (20). The number of calculations for $y_{i}$ is ten. Therefore, the calculation with the CGM is still quite complicated.

\section{Stiffness Decomposition Method}

If the SDM is applied to compute the displacement of the simply supported beam, the beam should be resegmented. The segments should be renumbered due to the corresponding division of the linear $\bar{M}(x)$ diagram at point $O$. This is different from the previous case. Correspondingly, the beam is divided into two parts: Part 1 and Part 2. There are five segments, \#1 through \#5, as shown in Figure 15.

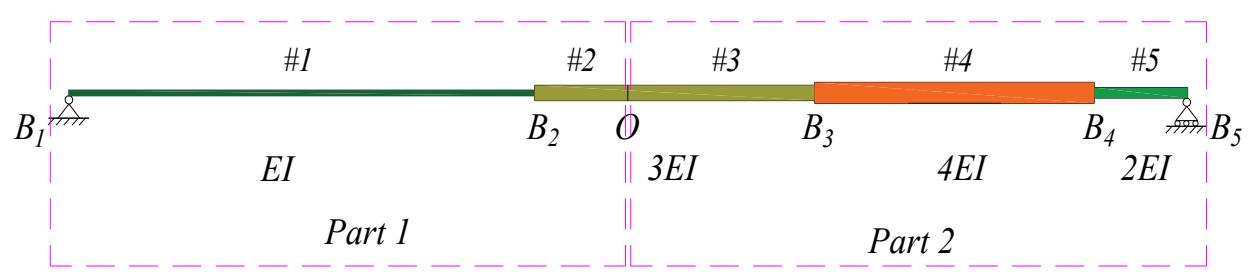

Figure 15. Segment renumbering of the simply supported beam.

To conveniently apply the SDM, we can compare the simply supported beam here with the general $n$-segment complex bending structure in Figure 1. It is obvious that $m_{1}=1, m_{2}=3, m_{3}=3, m_{4}=4$, and $m_{5}=2$ based on the basic 
principle of the method. Following the operation principle presented in Table 2, the stiffness decomposition flows corresponding to Part 1 and Part 2 of the simply supported structure are established in Table 6 and Table 7, respectively.

Table 6. Stiffness decomposition of Part 1 of the simply supported beam.

\begin{tabular}{|c|c|c|c|}
\hline \multirow[b]{2}{*}{ Segment } & \multicolumn{2}{|c|}{$\begin{array}{l}\text { Stiffness corresponding to each state of stiffness } \\
\text { decomposition }\end{array}$} & \multirow{2}{*}{$\begin{array}{l}\text { Stiffness in initial state } \\
\\
\text { Reciprocal of initial } \\
\text { stiffness (1) + (2) }\end{array}$} \\
\hline & $\begin{array}{c}\text { Reciprocal of the } 1^{\text {st }} \\
\text { postdecomposition stiffness } \\
\text { in the } 1^{\text {st }} \text { state }\end{array}$ & $\begin{array}{c}\text { Reciprocal of the } 2^{\text {nd }} \\
\text { postdecomposition stiffness } \\
\text { in the } 2^{\text {nd }} \text { state (2) }\end{array}$ & \\
\hline$\# 1$ & $1 / E I$ & $1 / \infty E I$ & $1 / E I$ \\
\hline$\# 2$ & $1 / E I$ & $-1 / 1.5 E I$ & $1 / 3 E I$ \\
\hline $\begin{array}{c}\text { Graph } \\
\text { multiplication }\end{array}$ & Figure 16 (a) & Figure $16(b)$ & / \\
\hline Displacement & $\Delta_{l}($ Equation $(8))$ & $\Delta_{2}($ Equation $(8))$ & $\Delta_{1}+\Delta_{2}($ Equation $(9))$ \\
\hline
\end{tabular}


Table 7. Stiffness decomposition of Part 2 of the simply supported beam.

\begin{tabular}{|c|c|c|c|c|}
\hline \multirow[b]{2}{*}{ Segment } & \multicolumn{3}{|c|}{ Stiffness corresponding to each state of stiffness decomposition } & \multirow{2}{*}{$\begin{array}{c}\text { Stiffness in } \\
\text { initial state } \\
\\
\text { Reciprocal of } \\
\text { initial stiffness } \\
\text { (1) }+ \text { (2) }+ \text { (3) }\end{array}$} \\
\hline & $\begin{array}{l}\text { Reciprocal of the } 3^{\text {rd }} \\
\text { postdecomposition } \\
\text { stiffness in the } 3^{\text {rd }} \\
\text { state (1) }\end{array}$ & $\begin{array}{l}\text { Reciprocal of the } 4^{\text {th }} \\
\text { postdecomposition } \\
\text { stiffness in the } 4^{\text {th }} \\
\text { state }\end{array}$ & $\begin{array}{l}\text { Reciprocal of the } 5^{\text {th }} \\
\text { postdecomposition } \\
\text { stiffness in the } 5^{\text {th }} \\
\text { state }\end{array}$ & \\
\hline \#3 & $1 / 3 E I$ & $1 / \infty 0 E I$ & $1 / \infty E I$ & $1 / 3 E I$ \\
\hline \#4 & $1 / 3 E I$ & $-1 / 12 E I$ & $1 / \infty E I$ & $1 / 4 E I$ \\
\hline$\# 5$ & $1 / 3 E I$ & $-1 / 12 E I$ & $1 / 4 E I$ & $1 / 2 E I$ \\
\hline $\begin{array}{c}\text { Graph } \\
\text { multiplication }\end{array}$ & Figure $16(\mathrm{c})$ & Figure 16 (d) & Figure $16(\mathrm{e})$ & l \\
\hline Displacement & $\Delta_{3}($ Equation $(8))$ & $\Delta_{4}($ Equation $(8))$ & $\Delta_{5}($ Equation $(8))$ & $\begin{array}{c}\Delta_{3}+\Delta_{4}+\Delta_{5} \\
\text { (Equation (9)) }\end{array}$ \\
\hline
\end{tabular}

After the stiffness decomposition, graph multiplication was conducted for each state of stiffness decomposition. These graph multiplications are illustrated in Figure 16 in accordance with the above stiffness decomposition flows in Tables 6 and 7. 


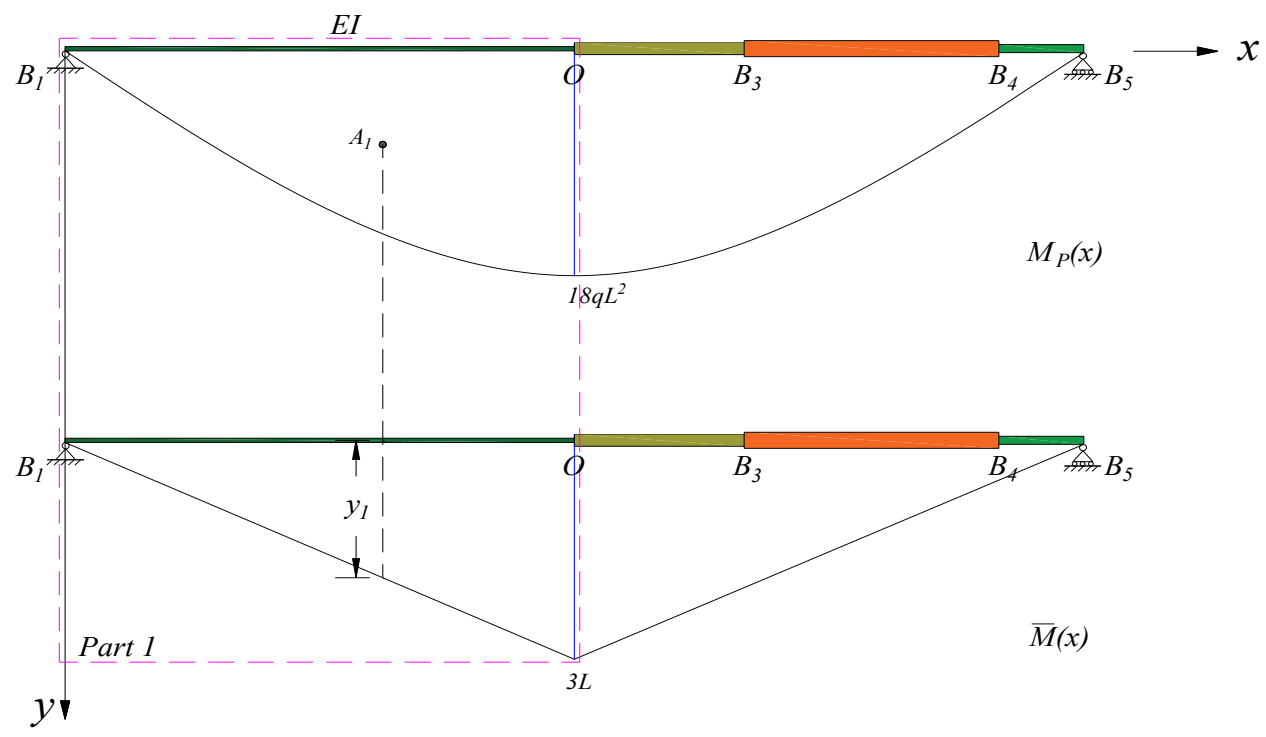

(a) $\Delta_{1}$ of the $1^{\text {st }}$ state for Part 1

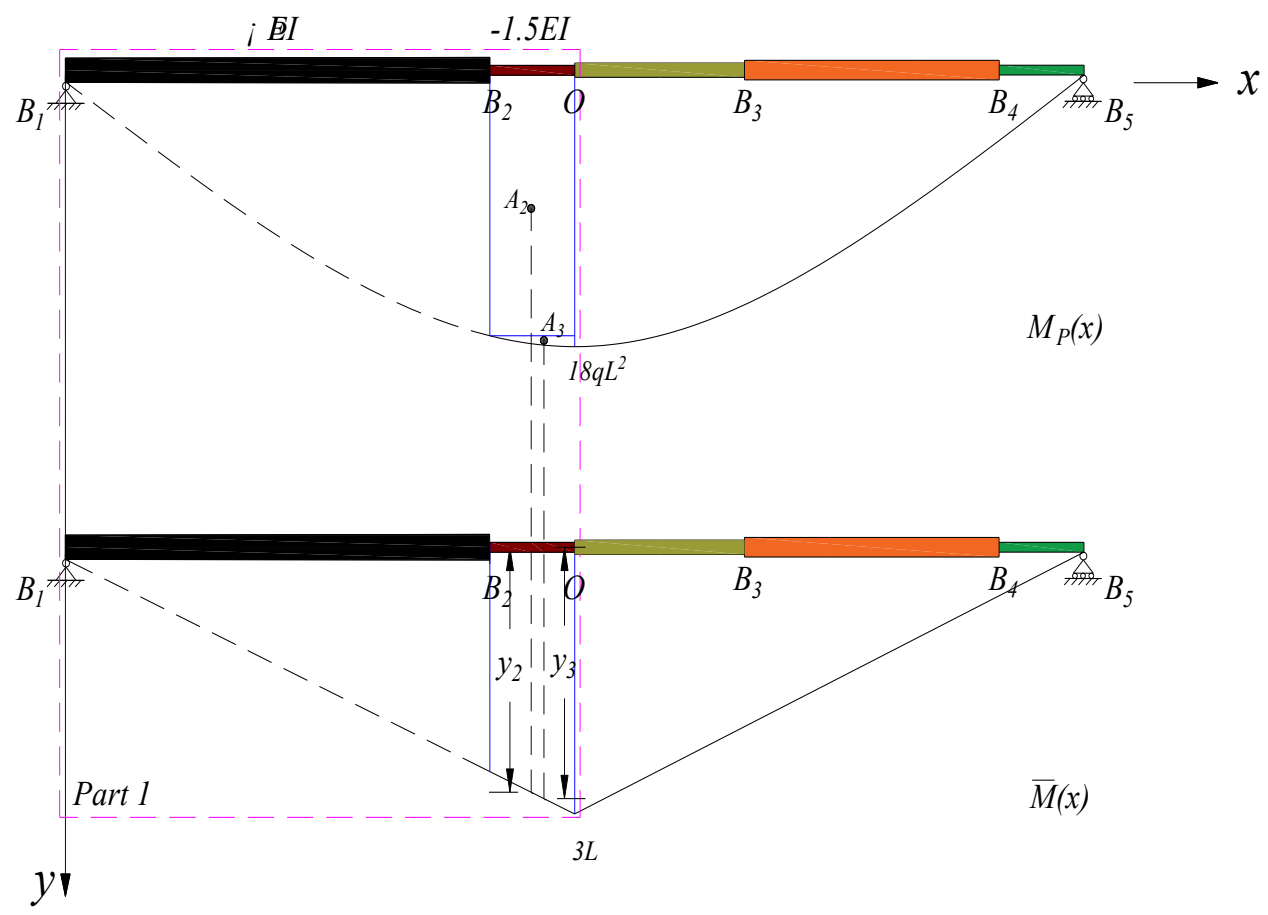

(b) $\Delta_{2}$ of the $2^{\text {nd }}$ state for Part 1 


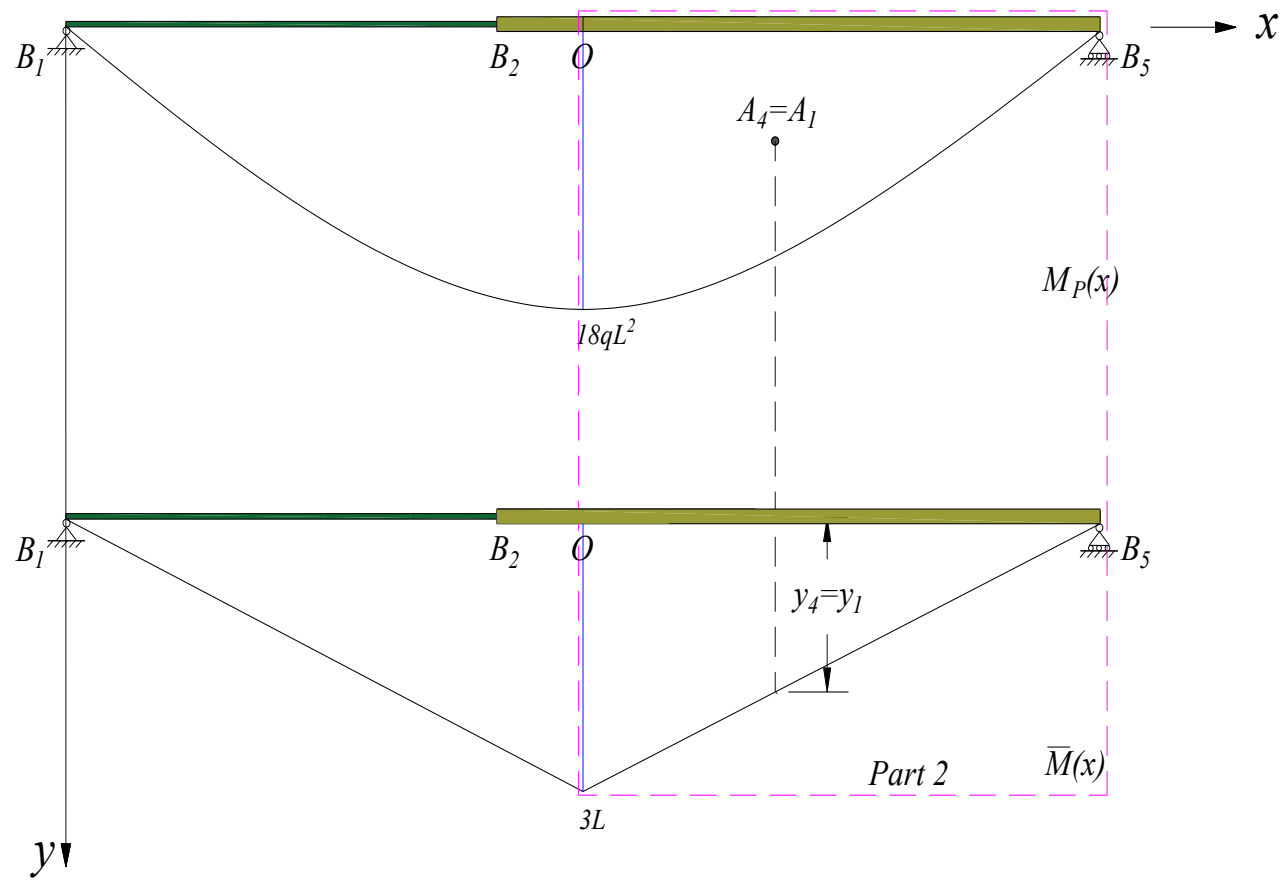

(c) $\Delta_{3}$ of the $3^{\text {rd }}$ state for Part 2

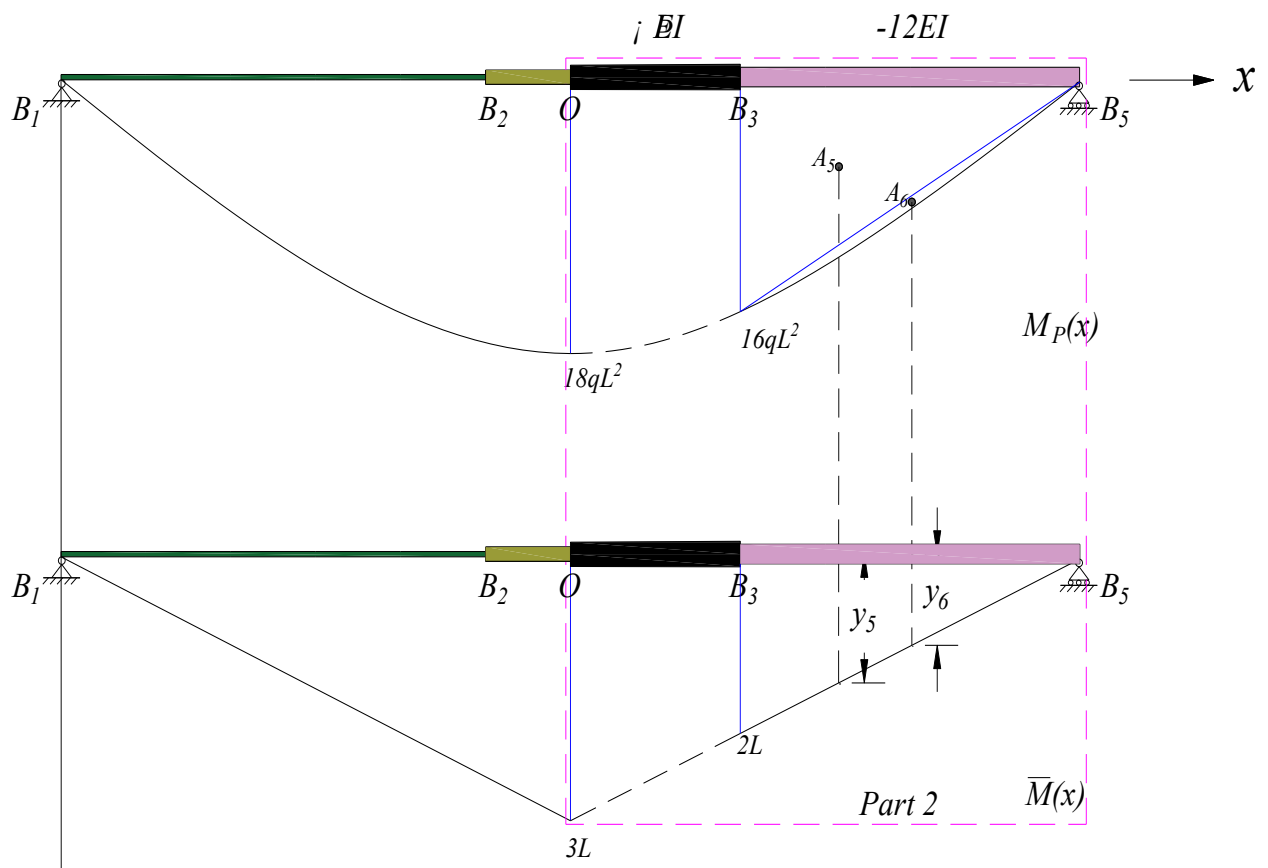


(d) $\Delta_{4}$ of the $4^{\text {th }}$ state for Part 2

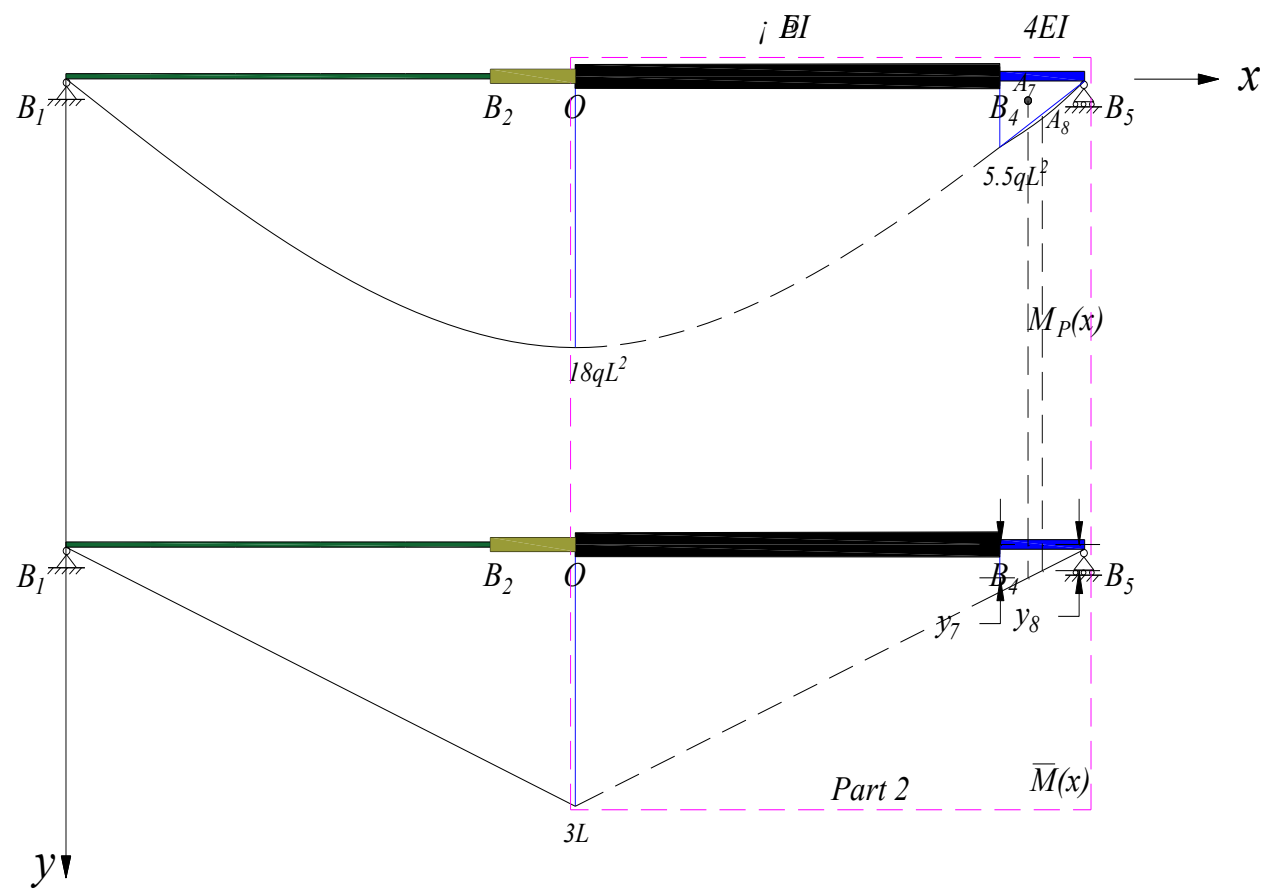

(e) $\Delta_{5}$ of the $5^{\text {th }}$ state for Part 2

Figure 16. Graph multiplication of each state for the simply supported beam via the SDM.

In Figure 16, we need only consider the graph multiplication of the solid-line $M_{P}(x)$ diagrams for the simply supported beam, which is similar to the first case. Due to the complexity of the structure, block division had to be conducted for these solid-line diagrams, as shown in Figure 16. Then, all the blocks in these figures were standard blocks. Their areas and the vertical coordinates of the linear bending moment $\bar{M}(x)$ diagrams were determined through comparisons with those in Table 1 . The results are provided in

$$
\begin{aligned}
& A_{1}=72 q L^{3}, \quad y_{1}=\frac{15 L}{8} ; \quad A_{2}=\frac{35 q L^{3}}{2}, \quad y_{2}=\frac{11 L}{4} ; \\
& \left\{A_{3}=\frac{q L^{3}}{3}, \quad y_{3}=\frac{45 L}{16} ; \quad A_{4}=A_{1}, \quad y_{4}=y_{1} ; \quad A_{5}=32 q L^{3}, \quad y_{5}=\frac{4 L}{3}\right. \text {; } \\
& A_{6}=\frac{16 q L^{3}}{3}, \quad y_{6}=L ; \quad A_{7}=\frac{11 q L^{3}}{4}, \quad y_{7}=\frac{L}{3} ; \quad A_{8}=\frac{q L^{3}}{12}, \quad y_{8}=\frac{L}{4}
\end{aligned}
$$

Equation (9) was implemented to calculate the displacement of the simply supported beam, as shown in 


$$
\begin{aligned}
D_{O} & =\hat{\mathrm{A}}_{i=1}^{5} D_{i} \\
& =\frac{1}{m_{1} E I} A_{1} y_{1}+\underset{i=2}{\mathrm{~A}} \frac{m_{i-1}-m_{i}}{m_{i-1} m_{i} E I} A_{i} y_{i} \\
& =\frac{1}{E I} A_{1} y_{1}-\frac{1}{1.5 E I}\left(A_{2} y_{2}+A_{3} y_{3}\right)+\frac{1}{3 E I} A_{4} y_{4}-\frac{1}{12 E I}\left(A_{5} y_{5}+A_{6} y_{6}\right)+\frac{1}{4 E I}\left(A_{7} y_{7}+A_{8} y_{8}\right) \\
& =\frac{27557 q L^{4}}{192 E I}
\end{aligned}
$$

\section{COMPARISON AND DISCUSSION}

A comparison between Equation (22) and Equation (18) shows that the displacement of the simply supported

\begin{tabular}{|c|c|c|c|c|c|}
\hline Method & \multicolumn{4}{|c|}{ Number of mathematical operations } & Complexity \\
\hline \multirow{2}{*}{ SDM (1) } & $A_{i}$ (3) & $y_{i}$ (4) & Graph multiplication & $\begin{array}{c}\text { Total } \\
(3)+(4)+(5)\end{array}$ & Each operation \\
\hline & 7 & 7 & 7 & 21 & Simple \\
\hline \multirow{2}{*}{ DIM (2) } & $\begin{array}{c}\text { Single } \\
\text { integration } \\
\text { 6 }\end{array}$ & $\begin{array}{c}\text { Operation step } \\
\text { per integration } \\
(7)\end{array}$ & / & $\begin{array}{l}\text { Total } \\
\text { (6) } \times(7)\end{array}$ & Each operation \\
\hline & 5 & 5 & I & 25 & Complicated \\
\hline (1)/(2) & l & I & I & $84.0 \%$ & / \\
\hline
\end{tabular}
beam calculated by the SDM is the same as that by the DIM. From the perspective of the total number of mathematical operations, a comparison between the two methods is shown in Table 8.

Table 8. Comparison between the SDM and DIM for the simply supported beam.

Table 8 shows that the total number of operations in the SDM accounts for $84.0 \%$ of those in the DIM. Moreover, each operation in the new method is very simple.

A comparison between Equation (22) and Equation (20) shows that the SDM can give the same displacement result as the CGM for the simply supported structure. Considering the complexity of the graph multiplication process, a comparison between the SDM and CGM is shown in Table 9. 
Table 9. Comparison between the SDM and CGM for the simply supported beam.

\begin{tabular}{|c|c|c|c|c|}
\hline \multirow{2}{*}{ Method } & \multicolumn{5}{|c|}{ Number of mathematical operations } \\
\cline { 2 - 5 } & $\mathbf{A}_{\mathbf{i}}(3)$ & $\mathbf{y}_{\mathbf{i}}(4)$ & Graph multiplication (5) & Total (3)+(4)+(5) \\
\hline SDM (1) & 7 & 7 & 7 & 21 \\
\hline CGM (2) & 11 & 10 & 11 & 32 \\
\hline (1)/(2) & $63.6 \%$ & $70.0 \%$ & $63.6 \%$ & $65.6 \%$ \\
\hline
\end{tabular}

Table 9 shows that the SDM only accounts for $65.6 \%$ of CGM in terms of the total number of mathematical operations for the displacement calculation of the simply supported beam. Obviously, these proportions will further decrease as the number of segments in the structure increases.

Therefore, the comparison of the three methods in the displacement calculation of the complex simply supported beam shows that the SDM is simpler and more efficient than the DIM and CGM.

\section{CONCLUSION}

Focusing on the disadvantages of the DIM and the CGM for displacement analytical solutions of complex bending beams, an innovative method called the SDM was proposed and developed to overcome the shortcomings of them. As an analytical method, the fundamental principle of the SDM was derived based on the unit load method. In the circumstance of rapid development of finite element technology recently, new analytical approaches have great significance in structural analysis. The general calculation equation of displacement was derived and provided for general $n$-segment complex bending structures. An operational procedure for the new method was constructed to facilitate its application. The clear mathematical and mechanical derivation of this method makes it easy to understand.

Then, the SDM was applied to some classic complex bending structures. The results verified the correctness and effectiveness of the proposed method. The application of the SDM to a 3-segment cantilever beam showed that the total number of computations required by the SDM accounted for only $47.4 \%$ to $60 \%$ of the number of computations required by the DIM and CGM. The application of the SDM to a 4-segment simply supported structure showed that the total number of computations required by the SDM accounted for $65.6 \%$ to $84.0 \%$ of the number of computations required by the DIM and CGM. It can be estimated that these proportions will further decrease as the number of segments in the structure increases. Moreover, the actual operations in the new method were simpler and more convenient than those in the other two methods. Therefore, the SDM can overcome the difficulties faced by the DIM and the CGM in solving the displacements of complex bending structures. Hence, the new method enables simpler, more efficient, and more practical displacement computations and simultaneously further develops the comparative advantages of the CGM over the DIM. The simplicity, practicality, and efficiency of this method make it have great potential to be applied extensively in structural engineering in the future. 


\section{REFERENCES}

Ren, M.Q., Gong, F.Y. \& Maekawa, K.C. 2019. Numerical investigation and design of reinforced concrete beams with non-uniform frost damage on the compression and tension sides. Structure and Infrastructure Engineering, 15(7): 983-999.

Huang, T., Yuan, Y., Zheng, J.L., Avital, E. \& Wen, P.H. 2019. Large deformations of tapered beam with finite integration method. Engineering Analysis with Boundary Elements, 107: 115-123.

Xia, Y., Liao, K. 2018. Locking-free isogeometric analysis of complex three-dimensional beam structures. Engineering Mechanics. 35(11): 17-25.

Tang, S. H., Chu, J. Q., Fang, Z., Zhang, X. B., Luo, C. F. 2019. Damage identification method of beam structure based on uniform load deflection curvature. Chinese Quarterly of Mechanics. 40(3): 549-559.

Ma, Y. X., Xu, W. H., Xu, Z. W. 2020. Displacement reconstruction of slender beam based on polynomial fitting method. Journal of Vibration and Shock. 39(11): 152-157, 183.

Wang, L, Wang, Z. C., Geng, D., Ren, W. X. 2017. Strain modes based dynamic deflection estimation of continuous beam structures. Engineering Mechanics. 34(1): 131-138.

Civalek, O., Demir, C. 2011. Bending analysis of microtubules using nonlocal Euler-Bernoulli beam theory. Applied Mathematical Modelling, 35(5): 2053-2067.

Eltaher, M.A., Emam, S. A., Mahmoud, F.F. 2012. Free vibration analysis of functionally graded size-dependent nanobeams. Applied Mathematics and Computation, 218: 7406-7420.

Demir, C., Civalek, O. 2013. Torsional and longitudinal frequency and wave response of microtubules based on the nonlocal continuum and nonlocal discrete models. Applied Mathematical Modelling, 37 (22): 9355-9367.

Akgöz, B., Civalek, O. 2014. Longitudinal vibration analysis for microbars based on strain gradient elasticity theory. Journal of Vibration and Control, 20 (4): 606-616.

Civalek, O., Uzun, B., Yaylı, MO., Akgöz, B. 2020. Size-dependent transverse and longitudinal vibrations of embedded carbon and silica carbide nanotubes by nonlocal finite element method. Eur. Phys. J. Plus, 135: 381.

Civalek, O., Demir, C. 2016. A simple mathematical model of microtubules surrounded by an elastic matrix by nonlocal finite element method. Applied Mathematics and Computation, 289: 335-352.

Shvab'yuk, V. I., Krutii, Yu. S. \& Sur'yaninov, M. G. 2016. Investigation of the free vibrations of bar elements with variable parameters using the direct integration method. Strength of Materials, 48 (3): 384-393.

Zeng, Z., Wu, Z.P., Luo, J., Yin, H.T., Li, P. \& Tu, Q.M. 2019. An analytical calculation method for displacement and force on continuous welded rails in temperature-transition zone. Construction and Building Materials, 207: 228-237.

Illouli, S., Sadaoui, A. \& Khennane A. 2019. Application of the theory statically indeterminate structures of infinite degree to a cable-truss footbridge under lateral forces. Engineering Structures, 188: 665-676.

Zhang, J.J., Da, L. 2018. Finite element calculation and standardization design of large-span steel roof truss based on unit load method. Engineering Journal of Wuhan University, 51(S1): 93-97.

Ghali, A. \& Neville, A. 2017. Structural Analysis: A Unified Classical and Matrix Approach. CRC Press and Taylor \& Francis Group, Boca Raton London New York. Pp.

Sun, Z.W. \& Wu, X.G. 2017. Optimization for hanger forces of tied-arch bridges based on stress balanced method. Engineering Journal of Wuhan University, 50(5): 704-707,713. 
Karnovsky, I.A. \& Lebed, O. 2011. Advanced Methods of Structural Analysis. Springer, New York Dordrecht Heidelberg

London. Pp.

Su, D.L., Zhang, L. \& Fan, Z.L. 2013. Analysis of Displacement of the Curved Bar Structure Based on Diagram Multiplication. Journal of Shenyang Jianzhu University (Natural Science), 29(3): 496-500.

Zhang, D.P., Cheng, W.M. \& Cai K. 2015. Deflection prediction for large tonnage gantry crane girder midspan base on diagram multiplication method. Mechanical Science and Technology for Aerospace Engineering, 34(10): 1568-1573.

Zheng, Y.G., Wang, Y. \& Song, Y.L. 2019. Principle and application of stiffness-disttribution diagram multiplication method. Mechanics in Engineering, 41(2): 227-232.

Huang, L., Ma, J., Deng, Y.H. \& Wang, B. 2016. Computing skills of force method for statically indeterminate beams with variable cross sections. Mechanics in Engineering, 38(4): 459-461.

Bao S.H. \& Gong, Y.Q. 2008. Structural Mechanics. Wuhan University of Technology Press, Wuhan. Pp.

Wu, Y.P., Zeng, Q., Han, B. \& Miao, Q.S. 2017. Research and engineering application on elastic deflection algorithm of steel beam. Building Structure, 47(S2): 207-210. 